ARTICLE

https://doi.org/10.1038/s41467-019-09524-z

\title{
Hepatocyte-specific loss of GPS2 in mice reduces non-alcoholic steatohepatitis via activation of PPAR $\alpha$
}

Ning Liang ${ }^{1}$, Anastasius Damdimopoulos ${ }^{1}$, Saioa Goñi ${ }^{1}$, Zhiqiang Huang ${ }^{1}$, Lise-Lotte Vedin (i) ${ }^{2}$, Tomas Jakobsson ${ }^{2}$, Marco Giudici', Osman Ahmed (1) 2, Matteo Pedrelli², Serena Barilla1, Fawaz Alzaid (1) ${ }^{3}$, Arturo Mendoza4, Tarja Schröder ${ }^{2}$, Raoul Kuiper ${ }^{2}$, Paolo Parini2 ${ }^{2,5,6}$, Anthony Hollenberg ${ }^{4}$, Philippe Lefebvre (1) ${ }^{7}$, Sven Francque (10) ${ }^{8,9}$, Luc Van Gaal ${ }^{9,10}$, Bart Staels ${ }^{7}$, Nicolas Venteclef ${ }^{3}$, Eckardt Treuter $^{1}$ \& Rongrong Fan ${ }^{1}$

Obesity triggers the development of non-alcoholic fatty liver disease (NAFLD), which involves alterations of regulatory transcription networks and epigenomes in hepatocytes. Here we demonstrate that G protein pathway suppressor 2 (GPS2), a subunit of the nuclear receptor corepressor (NCOR) and histone deacetylase 3 (HDAC3) complex, has a central role in these alterations and accelerates the progression of NAFLD towards non-alcoholic steatohepatitis (NASH). Hepatocyte-specific Gps2 knockout in mice alleviates the development of diet-induced steatosis and fibrosis and causes activation of lipid catabolic genes. Integrative cistrome, epigenome and transcriptome analysis identifies the lipid-sensing peroxisome proliferator-activated receptor $\alpha$ (PPAR $\alpha$, NR1C1) as a direct GPS2 target. Liver gene expression data from human patients reveal that Gps2 expression positively correlates with a $\mathrm{NASH}$ /fibrosis gene signature. Collectively, our data suggest that the GPS2-PPAR $\alpha$ partnership in hepatocytes coordinates the progression of NAFLD in mice and in humans and thus might be of therapeutic interest.

\footnotetext{
${ }^{1}$ Department of Biosciences and Nutrition, Karolinska Institutet, Huddinge 14157, Sweden. ${ }^{2}$ Department of Laboratory Medicine, Karolinska Institutet, Huddinge 14157, Sweden. ${ }^{3}$ INSERM, Cordeliers Research Centre, Sorbonne Paris Cité, Université Paris Descartes, Université Paris Diderot, Paris 75013 , France. ${ }^{4}$ Division of Endocrinology, Diabetes and Metabolism, Joan \& Sanford I. Weill Department of Medicine, Weill Cornell Medicine, New York 10021, USA. ${ }^{5}$ Department of Medicine Huddinge, Karolinska Institutet, Huddinge 14157, Sweden. ${ }^{6}$ Inflammation and Infection Theme, Karolinska University Hospital, Huddinge 14157, Sweden. ${ }^{7}$ University Lille, INSERM, CHU Lillie, Institut Pasteur de Lille, U1011-EGID, Lille F-59000, France. ${ }^{8}$ Department of Gastroenterology and Hepatology, University of Antwerp, Antwerp 2610, Belgium. ${ }^{9}$ Laboratory of Experimental Medicine and Pediatrics, University of Antwerp, Antwerp 2610, Belgium. ${ }^{10}$ Department of Endocrinology, Diabetology and Metabolism, University of Antwerp, Antwerp 2610, Belgium. Correspondence and requests for materials should be addressed to E.T. (email: eckardt.treuter@ki.se) or to R.F. (email: rongrong.fan@ki.se)
} 
$\mathrm{N}$ on-alcoholic fatty liver disease (NAFLD) is a chronic liver metabolic disorder which affects up to $30 \%$ of the adult population ${ }^{1}$. The severity of NAFLD ranges from simple steatosis to more severe stages of non-alcoholic steatohepatitis (NASH), characterized by liver inflammation, cell ballooning, and apoptosis $^{1,2}$. Multiple factors such as lipotoxicity, insulin resistance, and inflammation act in parallel to trigger the disease development ${ }^{1-3}$. Progress in understanding the role of promoting factors during disease development has revealed putative therapeutic targets including transcription factors (TFs), such as farnesoid X receptors (FXRs) ${ }^{4}$, liver X receptors (LXRs) ${ }^{5}$, thyroid hormone receptor (TR) $\beta^{6}$, and peroxisome proliferator-activated receptors (PPARs) ${ }^{7}$. Further targets are lipid-modulating and glucose-modulating enzymes, such as diglyceride acyltransferase $(\text { DGAT })^{8}$, Acetyl-CoA carboxylase (ACC) ${ }^{9}$, fatty acid synthase $(\text { FASN })^{10}$, AMP-activated protein kinase (AMPK) $)^{11}$, and metabolic hormones, such as incretins ${ }^{12}$ and fibroblast growth factors (FGFs) ${ }^{13,14}$. Although gene expression (transcriptome) analysis during NAFLD progression ${ }^{15,16}$ has identified a variety of differentially expressed marker genes, it has remained difficult to demonstrate whether differential expression is also the cause of disease progression in humans. NASH therapy remains challenging, in part due to the lack of understanding of the underlying molecular events that control those changes ${ }^{1-3}$.

Genetically modified mice and genome-wide sequencing approaches have revealed an intrinsic relationship between transcriptomes (gene expression patterns) and epigenomes (chromatin modifications) that is directly linked to the action of diverse coregulators $^{2,17-19}$. In contrast to well-studied TFs, the (patho-) physiological role of coregulators and epigenome alterations for the development of fatty liver disease remains to be explored. Notably, alterations in the expression and function of the histone deacetylase 3 (HDAC3) corepressor complex in macrophages and adipocytes have been linked to metabolic-inflammatory diseases, such as obesity and type 2 diabetes in humans ${ }^{20-22}$. In addition to HDAC3, structural core subunits of the complex include nuclear receptor corepressor (NCOR) (also NCOR1, N-CoR), silencing mediator of retinoid and TRs (SMRT, also NCOR2), and G protein pathway suppressor 2 (GPS2), all three of which have been originally identified as nuclear receptor-binding proteins, and the transducing beta-like proteins TBL1 and TBLR1 serving as coregulator exchange factors ${ }^{21,23-25}$. Previous studies have demonstrated that removal of NCOR, SMRT, and HDAC3 from hepatocytes in knockout (KO) mice results in increased steatosis due to disturbed lipid metabolic pathways and circadian signaling governed by TRs ${ }^{26,27}, \mathrm{LXRs}^{28}$, and Rev-Erbs ${ }^{29}$. PPAR-dependent genes were increased in the Ncor and Smrt KO models, supporting a role of these subunits in PPAR repression consistent with additional studies $28,30,31$. However, increased fatty acid oxidation upon removal of these subunits was not sufficient to reverse the steatosis phenotype driven by other nuclear receptor-dependent pathways including lipogenesis. One explanation could be that lipogenesis is necessary for the generation of endogenous PPARa ligands ${ }^{32}$. Surprisingly, TBL1 deficiency in hepatocytes resulted in reduced fatty acid oxidation, pointing at a PPARa-activating function of TBL1 independent of the corepressor complex ${ }^{33}$. The specific KO phenotypes of different subunits of what is thought to be the same corepressor complex suggests multiple target TFs to be affected, resulting in the modulation of in part opposing metabolic liver pathways, such as fatty acid oxidation versus lipogenesis. These data also raise the intriguing possibility that metabolic liver pathways that protect against NAFLD/NASH are controlled by a different corepressor subunit or sub-complex, the identity of which remains to be explored.

In this study, we discover a role of the GPS2 subunit in the progression of NAFLD/NASH in both mice and humans by combining the study of liver-specific Gps2 KO (LKO) mice with correlative analysis of human transcriptome datasets ${ }^{15,16}$. Through diet-induced mouse models of fatty liver disease along with next-generation sequencing analysis we provide evidence that Gps2 ablation improved liver steatosis and fibrosis, which correlated with the selective activation of lipid catabolic genes. By analyzing cistrome (GPS2 ChIP-seq), epigenome (H3K27ac, H3K4me3 ChIP-seq), and transcriptome (RNA-seq), we identified PPARa as a direct GPS2 target TF and verified the interplay of the two factors in single and double KO mice. Our analysis further reveals that GPS2 in hepatocytes functionally cooperates with NCOR but not with SMRT to repress PPARa. As these data collectively suggest that GPS2 promotes the progression of fatty liver disease via antagonizing PPARa, the selective therapeutic modulation of GPS2-PPARa interactions could be of interest for future disease interventions.

\section{Results}

Hepatocyte Gps2 depletion improves MCD-induced fibrosis in mice. We first explored the function of GPS2 in vivo using hepatocyte-specific Gps2 LKO mice (Fig. 1a). QPCR and western blot was performed to ensure the tissue-specific KO of Gps2 (Supplementary Fig. 1a-c). Gps2 LKO mice started to show reduced body weight comparing with WT mice at the age of 14 months old (Supplementary Fig. 1d). Gps2 LKO triglyceride in the very low-density lipoproteins (VLDL) fraction presented more than $50 \%$ reduction compared to WT mice, along with the total serum triglyceride level (Supplementary Fig. 1e). Lipoprotein triglyceride metabolism is tightly regulated via hepatic production and hydroxylation, but we found that serum (LPL) and hepatic lipoprotein lipase (HL) activities (Supplementary Fig. 1f) were similar between the two groups, suggesting decreased hepatic VLDL production rather than increased lipase activity. Intriguingly, Gps2 LKO mice showed more than 50\% increase in ketone body production in both fed and fasted state, suggesting a higher level of lipid oxidation in the LKO mice (Fig. 1b). This was further supported by enhanced oxygen consumption rate (OCR) in GPS2 knockdown AML12 cells both in basal and fatty acid abundant conditions (Fig. 1c). Notably, serum lipoprotein and intra-hepatic cholesterol levels were similar between WT and LKO mice (Supplementary Fig. 1g, h).

We further challenged the mice for 4 weeks with a fibrogenic methionine-deficient and choline-deficient diet (MCD). Both WT and LKO mice showed more than $30 \%$ of body weight loss yet no comparable difference between the two groups during the MCD feeding (Supplementary Fig. 1i). Liver expression of Gps 2 and Tbl1, but not of Ncor or Smrt, was increased upon MCD feeding for 2 and 4 weeks (Supplementary Fig. 1j). After 4 weeks MCD feeding, LKO mice showed a significant reduction of serum aspartate transaminase (AST) and alanine transaminase (ALT) activity, suggesting alleviated liver damage (Fig. 1d, e). Hematoxylin and eosin (HE) staining indicated reduced liver steatosis in LKO mice after MCD feeding (Fig. 1f), consistent with reduced liver triglyceride content (Fig. 1g). Serum lipoprotein triglyceride was not changed in MCD-fed mice (Supplementary Fig. 1k). Liver cholesterol levels were also not changed by Gps2 ablation (Supplementary Fig. 11). Consistently, Sirius red staining of the MCD-fed WT and LKO mice showed improved liver fibrosis in the LKO group upon MCD feeding (Fig. 1h, i), confirmed by hydroxyproline analysis (Fig. 1j).

As lipid-induced fibrosis progression is also highly related to liver inflammation ${ }^{34}$, we treated mice with lipopolysaccharide (LPS) and tested the pro-inflammatory gene expression in the livers (Supplementary Fig. 1m). While Il1b, Il6, Tnfo, Ccl2 and 

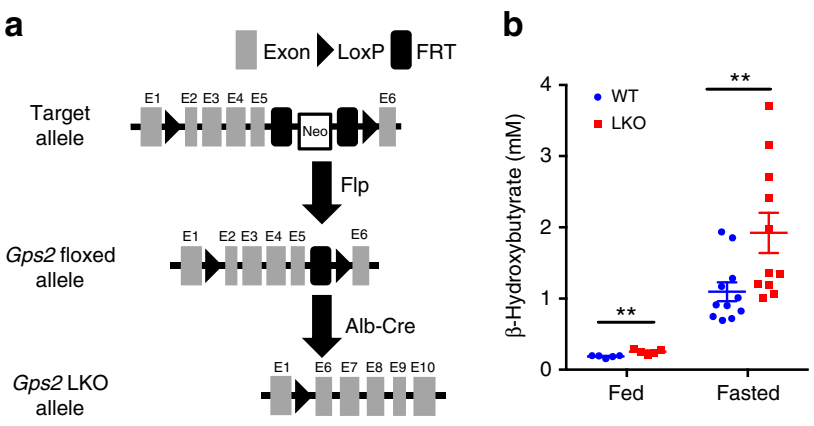
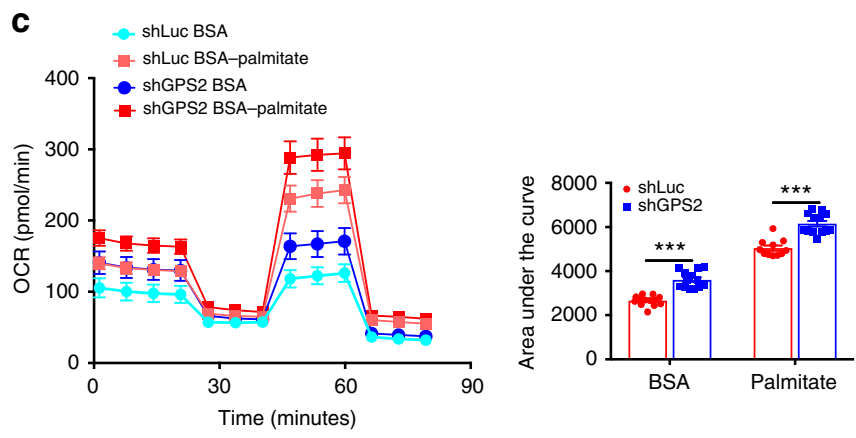

d

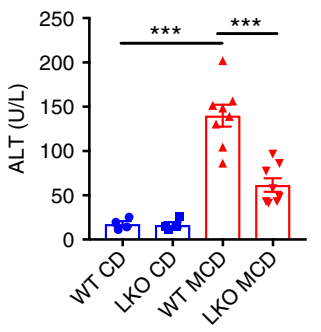

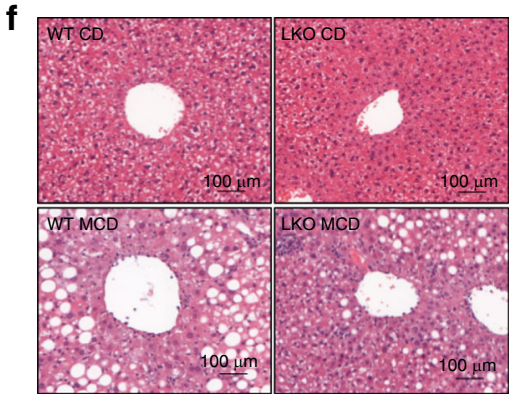

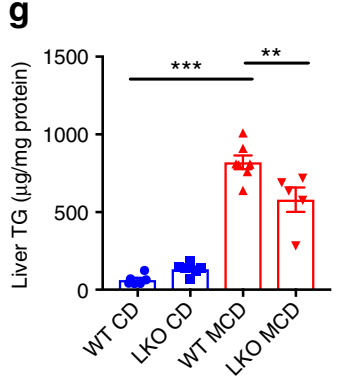

h
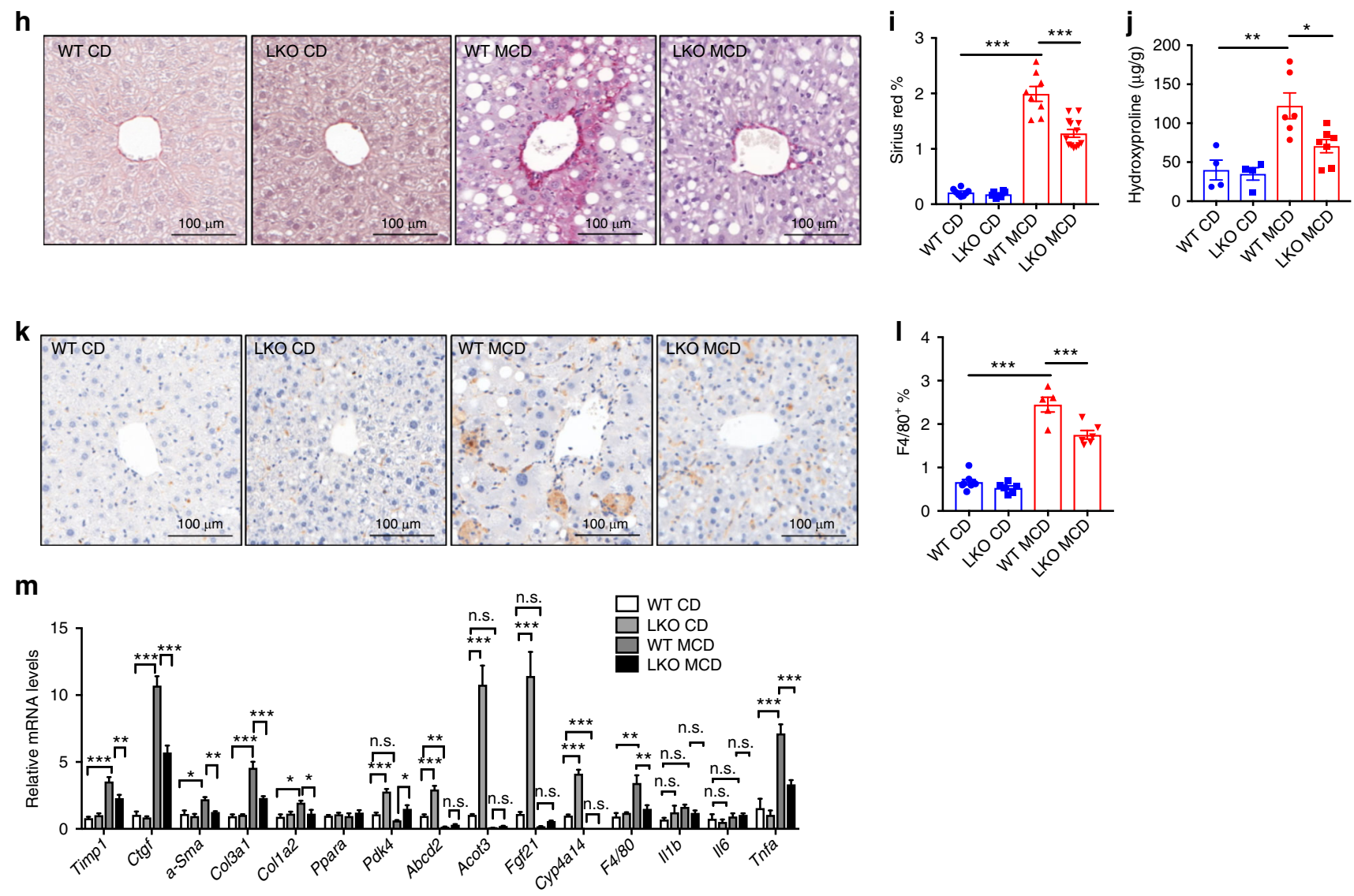

Fig. 1 Hepatocyte Gps2 depletion in mice improves MCD-induced fibrosis. a Flowchart representing the Gps2 floxed mice generation and LKO strategy. b Fed and fasted ketone body analysis, fed $n=5$, fasted $n=11$, one-way ANOVA followed by Tukey's test. c Mitochondrial respiration reflected by OCR levels in BSA or BSA-palmitate treated control (shLuc) and GPS2 knockdown (shGPS2) AML12 cell lines (left panel) and area under the curve calculations (right panel), $n=13$, one-way ANOVA followed by Tukey's test. (d) Serum ALT and (e) AST activity in chow diet (CD) and MCD-treated WT and LKO mice, $n=4$ in $C D$ and $n=8$ in MCD, one-way ANOVA followed by Tukey's test. (f) H\&E staining; (g) liver triglyceride, $n=6$ in WT CD, $n=7$ in LKO CD, $n=7$ in WT MCD and $n=5$ in LKO MCD; (h) surius red staining; (i) liver sirius red staining quantification, $n=8$ in WT CD, $n=6$ in LKO CD, $n=8$ in WT MCD, $n=13$ in LKO MCD; (j) liver hydroxyproline test, $n=4$ in CD groups, $n=6$ in WT MCD, $n=7$ in LKO MCD; (k) F4/80 staining; (I) F4/80 staining quantification, $n=8$ in WT CD, $n=6$ in LKO CD, $n=5$ in WT MCD, $n=6$ in LKO MCD; and (m) qPCR analysis of gene expression, $n=5-7$ in each groups, one-way ANOVA followed by Tukey's test. All data are represented as mean \pm s.e.m. ${ }^{\star} P<0.05,{ }^{\star \star} P<0.01,{ }^{\star \star \star} P<0.001$ 
$\mathrm{Ccl} 7$ were all induced by LPS, they showed no difference between the WT and LKO groups.

However, F4/80 staining demonstrated reduced macrophage infiltration in MCD-fed LKO mice compared with WT mice (Fig. 1k, l). QPCR analysis in CD and MCD mice showed reduced fibrotic and inflammation markers, along with increased fatty acid catabolic genes (Fig. $1 \mathrm{~m}$ ).

Lastly, genes related to lipid metabolism were already upregulated in LKO mice under basal conditions, while fibrosis markers remained unchanged (Supplementary Fig. 1n). These data collectively suggest that loss of GPS2 in hepatocytes regulates inflammation and fibrosis indirectly via regulating fatty acid metabolism.

Gps2 LKO improves liver steatosis and insulin resistance. We further explored the role of hepatocyte GPS2 in obesity-induced liver steatosis and insulin resistance. We first analyzed the phenotypes of mice upon chow diet feeding. Two-month-old chow diet-fed mice showed comparable blood glucose levels (Supplementary Fig. 2a), LKO mice had reduced fed and fasted (12 h fasting) blood glucose after 12 months (Supplementary Fig. 2b) and 16 months (Supplementary Fig. 2c). Fed insulin levels in both 2 months old (Supplementary Fig. 2d) and 16 months old (Supplementary Fig. 2e) mice were reduced as well, suggesting improved glucose control in LKO mice. Consistently, oral glucose tolerance test (OGTT, upon $12 \mathrm{~h}$ fasting) and insulin tolerance test (ITT, upon $4 \mathrm{~h}$ fasting) in 2 months old (Supplementary Fig. 2f, g) and 16 months old mice (Supplementary Fig. 2h, i) showed improved glucose control. We next proceeded to challenge the mice with high fat diet (HFD). Sixteen weeks of HFD increased Gps2 expression (Supplementary Fig. 2j). We found that HFD-induced body weight gain was significantly lower in LKO mice (Fig. 2a), while food and water intake remained similar (Supplementary Fig. 2k, 1). The subcutaneous fat was lower in LKO mice (Supplementary Fig. 2m). HFD-induced blood glucose levels were also significantly lower in LKO mice (Fig. 2b) although insulin levels were comparable (Fig. 2c). The OGTT and ITT test showed improved glucose control in HFD-fed LKO mice (Fig. 2d, e). Serum VLDL and total triglyceride levels (upon $4 \mathrm{~h}$ fasting), and HDL and total cholesterol levels were reduced in HFD LKO mice (Fig. 2f, Supplementary Fig. 2n). HE staining showed improved liver steatosis in LKO mice (Fig. $2 \mathrm{~g}$ ), confirmed by liver triglyceride analysis (Fig. 2h), although liver cholesterol level remained unchanged (Supplementary Fig. 2o). F4/80 staining showed slightly increased macrophage infiltration upon HFD treatment in WT mice (Fig. $2 \mathrm{i}, \mathrm{j}$ ), which was reduced in LKO mice (Fig. 2i, j). Gene expression analysis revealed reduced lipogenesis gene expression and increased fatty acid oxidation gene expression in the LKO mice liver (Fig. 2k). Il6 and Tnfa were reduced in LKO mice as well (Fig. 2k). In contrast, HFD did not significantly induce fibrotic markers in the liver in both WT and LKO mice (Supplementary Fig. 2p).

Taken together, the results suggest that hepatocyte Gps2 KO reduces body weight and plays a key role in modulating both lipid metabolism and glucose homeostasis in obese mice.

PPARa is a direct target of GPS2 in hepatocytes. We continued to investigate underlying mechanisms involved in the regulatory role of GPS2 in the liver. We first identified GPS2 signature genes from WT and LKO livers in chow diet fed mice using RNA-seq (Fig. 3a). KEGG pathway analysis revealed enrichment of upregulated genes in multiple metabolic pathways with PPAR pathways appeared in top enriched signaling pathways (Fig. 3b). Given the dominant role of PPARa in liver lipid oxidation ${ }^{35}$, we identified PPARa target genes in mouse livers using available transcriptome datasets ${ }^{36}$ (GSE73298 and GSE73299, Supplementary Fig. 3a), followed by a comparison of GPS2-regulated liver transcriptomes with both global (Supplementary Fig. 3b) and liver-specific Ppara KO mice (Supplementary Fig. 3c). We found that around $20 \%$ of the PPAR $\alpha$ target genes were regulated by GPS2 in both groups of comparisons, and among these more than $80 \%$ were repressed by GPS2 (Supplementary Fig. 3b, c). We additionally compared the PPARa target genes concluded from a previous report ${ }^{37}$. Among 121 target genes, 52 (including Cyp4a14, Pdk4, Fgf21) were up-regulated in Gps2 LKO livers, 27 were down-regulated and 42 remained unchanged (Supplementary Fig. 3d, e). Intriguingly, LXR agonist GW3965 treatment did not induce significant differences between WT and LKO mice (Supplementary Fig. 3f), suggesting GPS2 selectivity towards PPARa.

We next tested liver gene expression in fasted (Fig. 3c) and GW7647-treated mice (Fig. 3d). The results show that LKO mice had enhanced target gene responses to both treatments (Fig. 3c, d). We then determined the liver GPS2 cistrome using ChIP-seq. In agreement with the transcriptome data, TF binding motif analysis revealed PPAR-binding sites among the top GPS2occupied sites (Fig. 3e). Co-immunoprecipitation assays revealed that interaction with PPARa was dependent on the GPS2 region aa $100-155$ (Fig. 3f), part of the previously characterized nuclear receptor-binding domain ${ }^{38}$.

To demonstrate the requirement of PPARa for GPS2 repression of lipid metabolic genes, we generated Gps2 and Ppard double KO (PGKO) mice by cross-breeding the two single $\mathrm{KO}$ strains (Fig. 3g). PGKO and Ppara KO (PKO) mice had similar body weight (Supplementary Fig. 4a), liver weight (Supplementary Fig. 4b), liver cholesterol, and triglyceride levels (Supplementary Fig. 4c, d). Notably, qPCR showed that Gps2 ablation did not induce the PPARa target genes Pdk4, Cyp4a14, Fgf21 in PGKO livers that lack PPAR (Fig. 3g). Lipid oxidation seen in Gps2 LKO versus WT mice was not observed in PGKO versus PKO mice, as indicated by unchanged fed and fasted ketone body generation (Fig. 3h), suggesting that GPS2 repression of lipid oxidation was dependent on PPARa. Blood glucose (Supplementary Fig. 4e) and insulin levels (Supplementary Fig. 4f) were also not changed between the two groups. Above all, serum lipoprotein and triglyceride levels remained significantly increased in PKO mice, but were unchanged by Gps2 depletion (Supplementary Fig. 4g).

Collectively, these data identify PPARa as a major target TF for GPS2 in the liver.

GPS2 requires PPARa to modulate the hepatic epigenome. To define the GPS2-dependent cistrome and epigenome in mouse liver, we performed ChIP-seq of GPS2 along with H3K27ac and $\mathrm{H} 3 \mathrm{~K} 4 \mathrm{me} 3$ in WT and LKO mice (Fig. 4a). Alignment of the ChIP-seq peak heatmaps of H3K27ac, H3K4me3, and H3K4me1 (GSE29218) $^{39}$ with GPS2 revealed the presence of GPS2 in both active promoter and enhancer regions (Fig. 4a), which was confirmed by GPS2 peak distribution analysis (Supplementary Fig. 4h). Comparison of the GPS2-dependent transcriptome and epigenome revealed that transcriptional and epigenetic activation at GPS2-sensitive gene loci were highly coordinated (Supplementary Fig. 4i-n). Both $\mathrm{H} 3 \mathrm{~K} 27 \mathrm{ac}$ and $\mathrm{H} 3 \mathrm{~K} 4 \mathrm{me} 3$ levels are higher in LKO up-regulated (GPS2-repressed) genes compared to unchanged (GPS2-resistant) genes. Likewise, downregulated gene loci had lower H3K27ac and H3K4me3 levels (Supplementary Fig. 4i, j). Correlation analysis of GPS2-sensitive genes showed significant correlation with both $\mathrm{H} 3 \mathrm{~K} 27 \mathrm{ac}$ and $\mathrm{H} 3 \mathrm{~K} 4 \mathrm{me} 3$ levels (Supplementary Fig. 4k, 1), marking direct GPS2-mediated gene regulation via those promoters and enhancers (Supplementary 
a

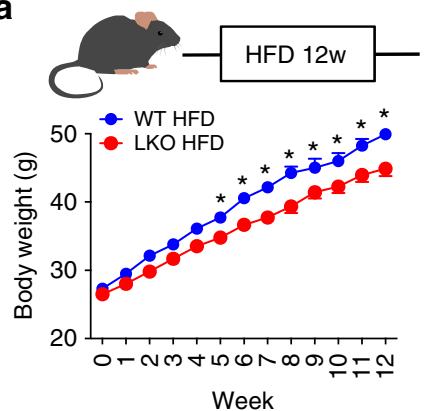

d

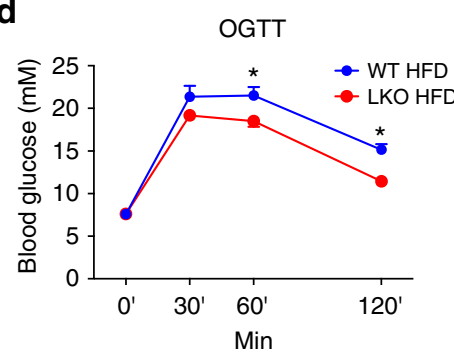

g
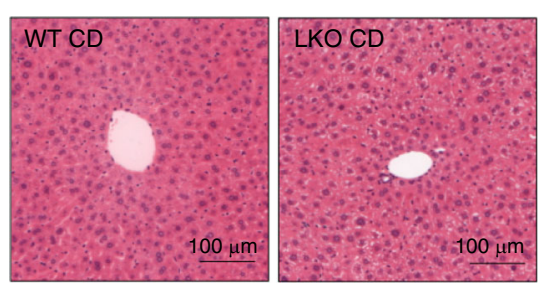

b

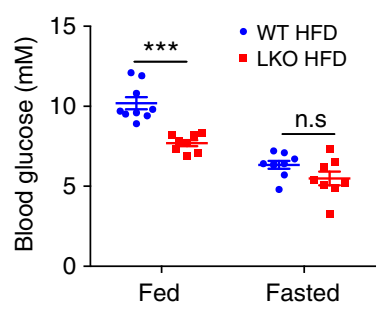

e

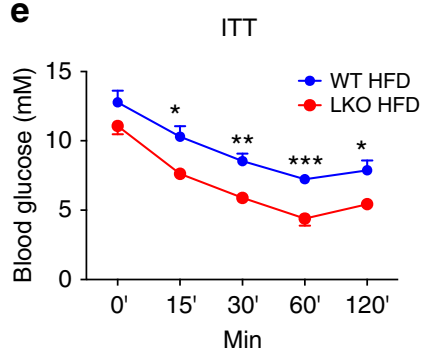

c

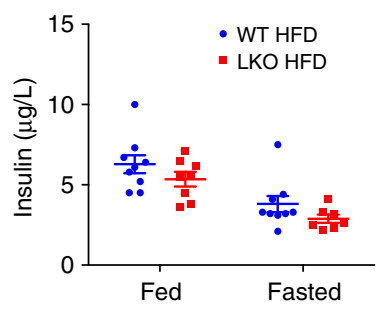

f

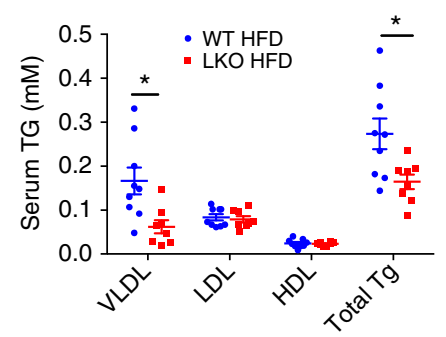

h

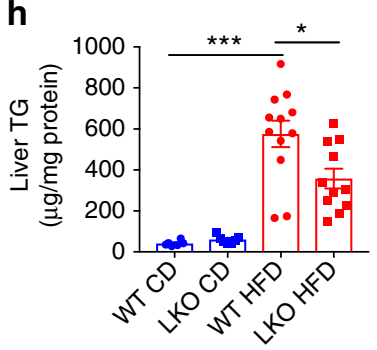

j

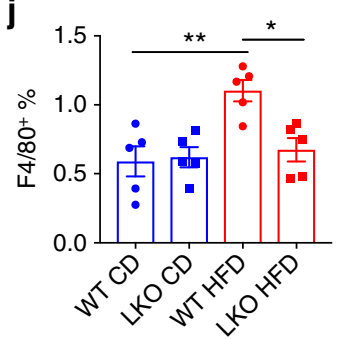

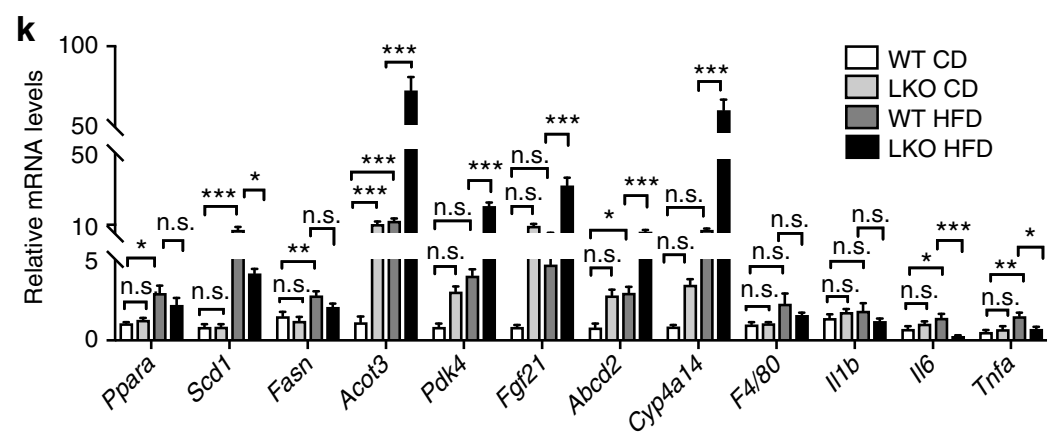

Fig. 2 Gps2 LKO mice are resistant to HFD. a Body weight change during 12 weeks HFD feeding in WT and LKO mice, $n=9-16$ in WT and $n=10-17$ in LKO, non-parametric Mann-Whitney test. Fed and fasted (b) glucose and (c) insulin in WT and LKO mice, $n=9$ in WT and $n=8$ in LKO, non-parametric Mann-Whitney test. (d) OGTT and (e) ITT in WT and LKO mice after 12 weeks of HFD, $n=10$, non-parametric Mann-Whitney test. (f) Triglyceride in VLDL fractions, $n=8-9$ in each group. non-parametric Mann-Whitney test. (g) H\&E staining; (h) liver triglyceride levels, $n=6$ in WT CD, $n=7$ in LKO $\mathrm{CD}, n=12$ in WT HFD and $n=11$ in LKO HFD, one-way ANOVA followed by Tukey's test. (i) F4/80 staining and (j) quantification, $n=5$ in each group. (k) QPCR analysis of liver mRNA expressions, $n=5-9$ in each group from 12 weeks CD-fed or HFD-fed WT and LKO mice, one-way ANOVA followed by Tukey's test. All data are represented as mean \pm s.e.m. ${ }^{\star} P<0.05,{ }^{\star \star} P<0.01,{ }^{\star \star \star} P<0.001$ 
a $\begin{array}{lll}-1.5 & 0 & 1.5\end{array}$
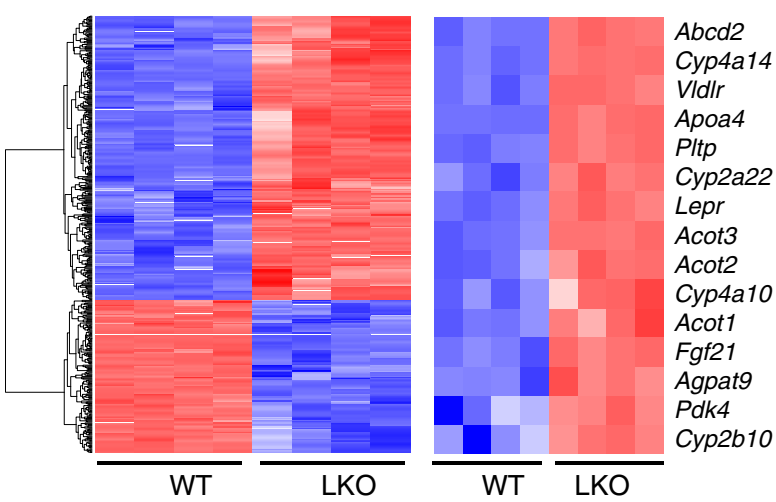

c

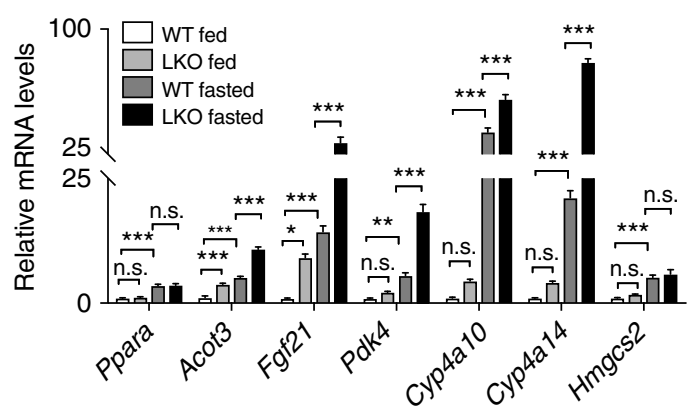

e b

\begin{tabular}{lcc}
$\begin{array}{l}\text { Upregulated genes in LKO }(\operatorname{LogFC}> \\
\text { Pathway }\end{array}$ & 1, Adj_P value $<0.05)$ \\
Gene & Statistics (Adj_P value) \\
\hline Arachidonic acid metabolism & 15 & $2.61 \mathrm{E}-07$ \\
Retinol metabolism & 12 & $8.58 \mathrm{E}-06$ \\
Biosynthesis of unsaturated fatty acids & 7 & $3.60 \mathrm{E}-05$ \\
Metabolic pathways & 52 & $2.00 \mathrm{E}-04$ \\
PPAR signaling pathway & 8 & $7.80 \mathrm{E}-03$
\end{tabular}

Downregulated genes in LKO $(\operatorname{LogFC}<-1$, Adj_Pvalue $<0.05)$

\begin{tabular}{lcc}
\multicolumn{1}{c}{ Pathway } & Gene & Statistics (Adj_P value) \\
\hline Steroid hormone biosynthesis & 7 & $2.25 \mathrm{E}-05$ \\
Prion diseases & 4 & $5.50 \mathrm{E}-03$ \\
Retinol matabolism & 5 & $6.60 \mathrm{E}-03$ \\
Ascorate and aldarate metabolism & 3 & $1.10 \mathrm{E}-02$
\end{tabular}

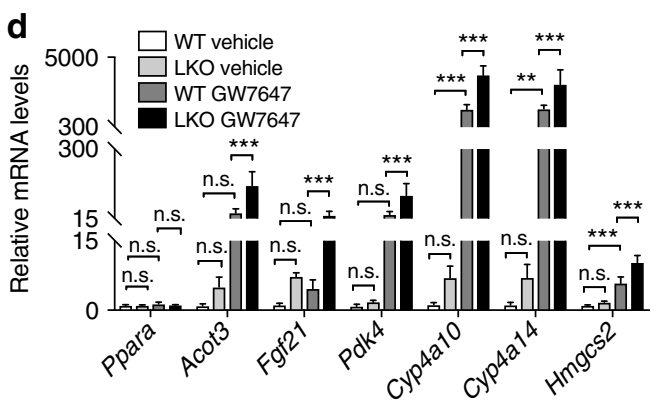

f
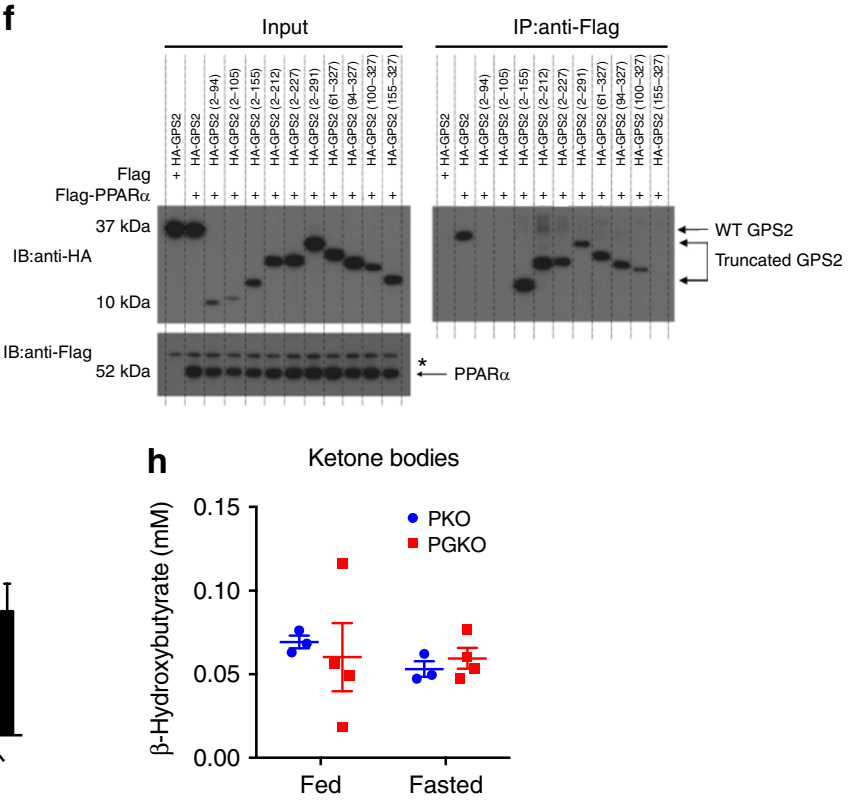

Fig. 3 GPS2 depletion causes PPAR $\alpha$ activation. a Heatmap representing the top 1000 significant (sorted based on adj $P$ value) gene expression in CD-fed WT and LKO livers. b KEGG pathway analysis of significantly up-regulated and down-regulated genes in LKO mice. c, $\mathbf{d}$ QPCR analysis of mRNA expression in WT and LKO mice upon (c) fasting, $n=5$ in each group; and (d) PPAR $\alpha$ agonist (GW7647) treatment, $n=5$ in the vehicle groups, $n=7$ in the GW7647-treated groups, one-way ANOVA followed by Tukey's test. e Motif analysis of GPS2-occupied regions in mouse liver. $\mathbf{f}$ Co-immunoprecipitation of GPS2 domains with PPAR $\alpha$ in 293 cells ( ${ }^{*}$ represents non-specific bands). $\mathbf{g}$ QPCR analysis of GPS2-regulated genes and (h) fed and fasted serum ketone bodies in PPAR $\alpha$ KO (PKO) and liver Gps2/Ppar $\alpha$ double KO (PGKO) mice livers, $n=3$ in PKO and $n=4$ in PGKO, non-parametric Mann-Whitney test. All data are represented as mean \pm s.e.m. ${ }^{\star} P<0.05,{ }^{\star \star} P<0.01,{ }^{\star \star \star} P<0.001$

Fig. $4 \mathrm{~m}, \mathrm{n})$, as represented for repression in the $P d k 4$ and Cyp4a14 loci (Fig. 4b, c).

We further compared the ChIP-seq profiles of GPS2 and PPAR $a$ in mouse livers. GPS2 and PPAR $a$ were co-localized in the Pdk4 and Cyp4a14 loci (Fig. 4d, e, GSE61817) ${ }^{40}$. Globally, around $85 \%$ of the GPS2 peaks were overlapped with PPARabinding sites (Fig. 4f). Moreover, GPS2 ablation increased H3K27ac in Pdk4 and Cyp4a14 promoter/enhancer loci, which 
a

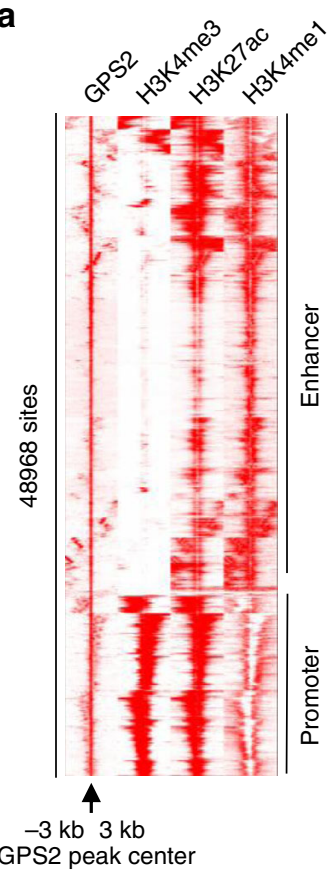

f

GPS2 peak center b

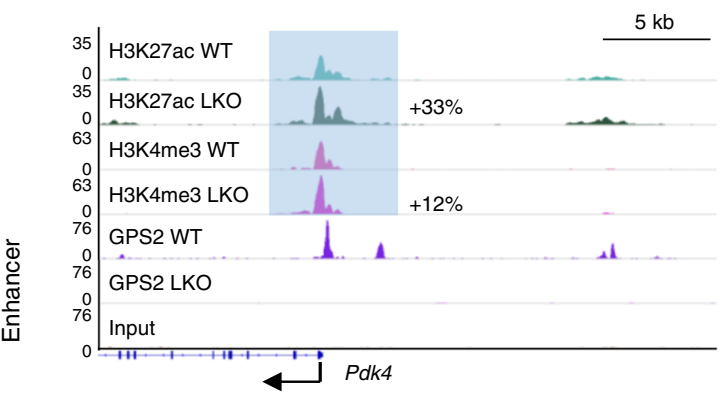

d

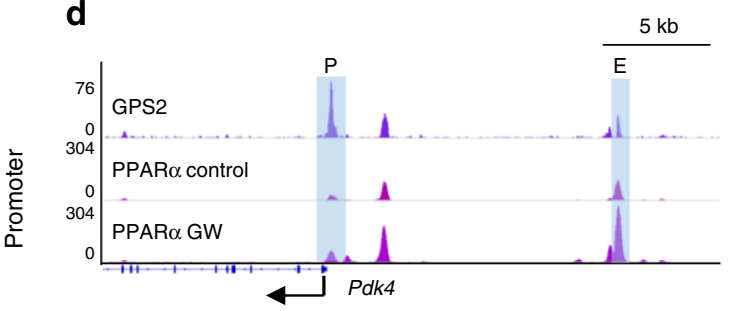

C

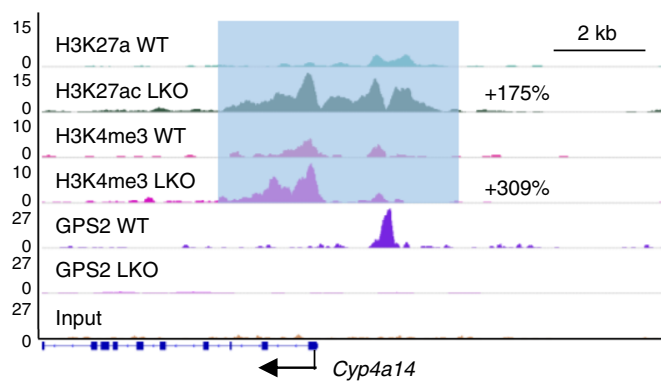

e

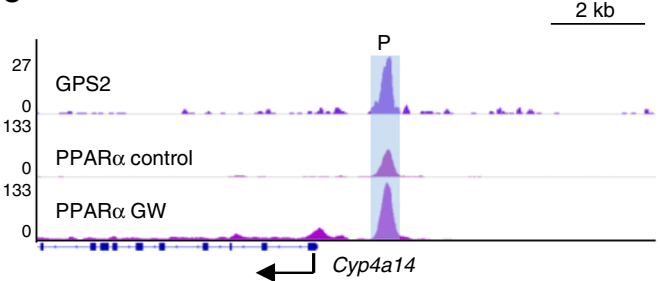

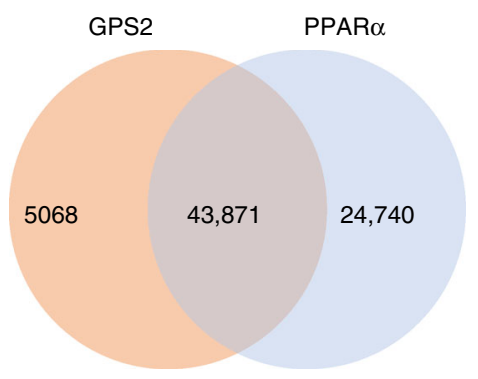

g
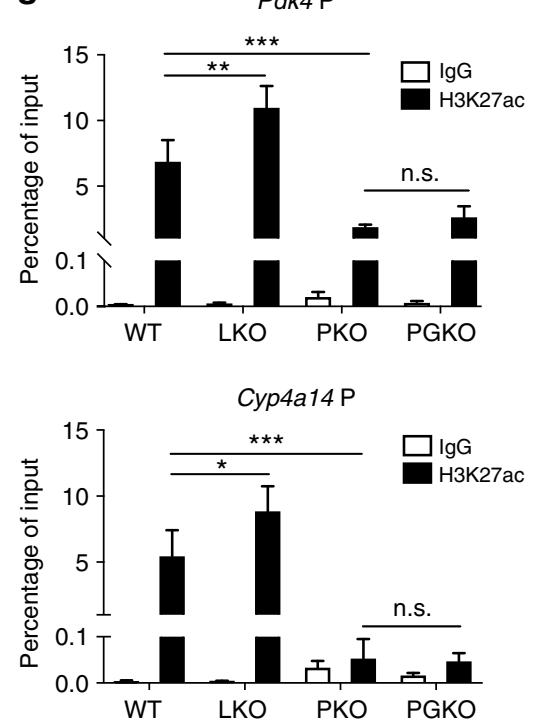

Pdk4 E

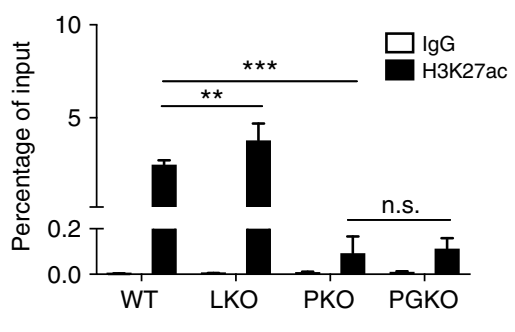

Control

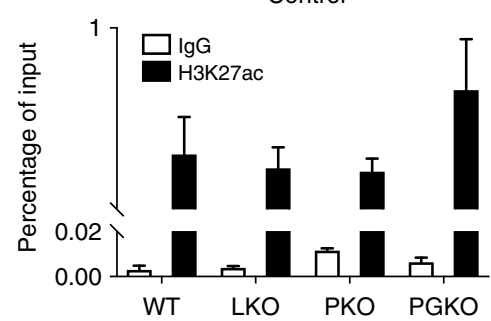

Fig. 4 GPS2 represses PPAR $\alpha$-regulated promoters and enhancers. a GPS2, H3K4me3, H3K27ac, and H3K4me1 ChIP-seq peaks are aligned to the GPS2 peak center in a window of $\pm 3 \mathrm{~kb}$. b, c ChIP-seq tracks of GPS2, H3K27ac, and H3K4me3 peaks at (b) Pdk4 and (c) Cyp4a14 loci, percentage of increase (LKO versus WT) represents logFC of each peak and is calculated from biological duplicates. d, e ChIP-seq tracks of GPS2, PPAR $($ vehicle and GW7647 treated, GSE61817) peaks at (d) Pdk4 and (e) Cyp4a14 loci. $\mathbf{f}$ Venn diagram representing the overlapped GPS2 and PPAR $\alpha$ peaks detected at least twice in mouse liver. $\mathbf{g}$ Further ChIP qPCR validation of H3K27ac at Pdk4 promoter (upper left), Pdk4 enhancer (upper right), Cyp4a14 promoter (lower left) and control (lower right) locus in WT, LKO, PKO, and PGKO livers, $n=5$ in WT and LKO groups, $n=4$ in PKO group and $n=3$ in PGKO group, one-way ANOVA followed by Tukey's test. All data are represented as mean \pm s.e.m. ${ }^{\star} P<0.05,{ }^{\star \star} P<0.01,{ }^{\star \star \star} P<0.001$

was not observed in PGKO versus PKO mice (Fig. 4g), suggesting that PPARa is required for GPS2-mediated epigenomic repression at those gene loci.

GPS2 cooperates with NCOR in hepatocytes. The apparent PPARa-selectivity of GPS2 repression was surprising as it was not seen in the comparable liver KO models for other complex subunits. Thus, we next investigated whether GPS2 functions within the corepressor complex to modulate liver gene expression. We first compared the chromatin recruitment of NCOR and SMRT with GPS2 in mouse livers. Cistrome analysis revealed that all three subunits shared more than $50 \%$ of all binding sites in the liver genome (Supplementary Fig. 5a, b, GSE26345 and GSE51045) ${ }^{29,41}$. Gps2 LKO livers shared overlapping transcriptome signatures with both Ncor and Smrt LKO livers 26,41 (Supplementary Fig. 5c, d). However, KEGG pathway analysis of the genes co-repressed by GPS2 and NCOR revealed PPAR signaling in the top list, along with liver metabolic pathways (Supplementary Fig. 5e), which was not observed for the genes corepressed by GPS2 and SMRT (Supplementary Fig. 5f). At the epigenome level, H3K27ac levels were similarly increased in Gps2 

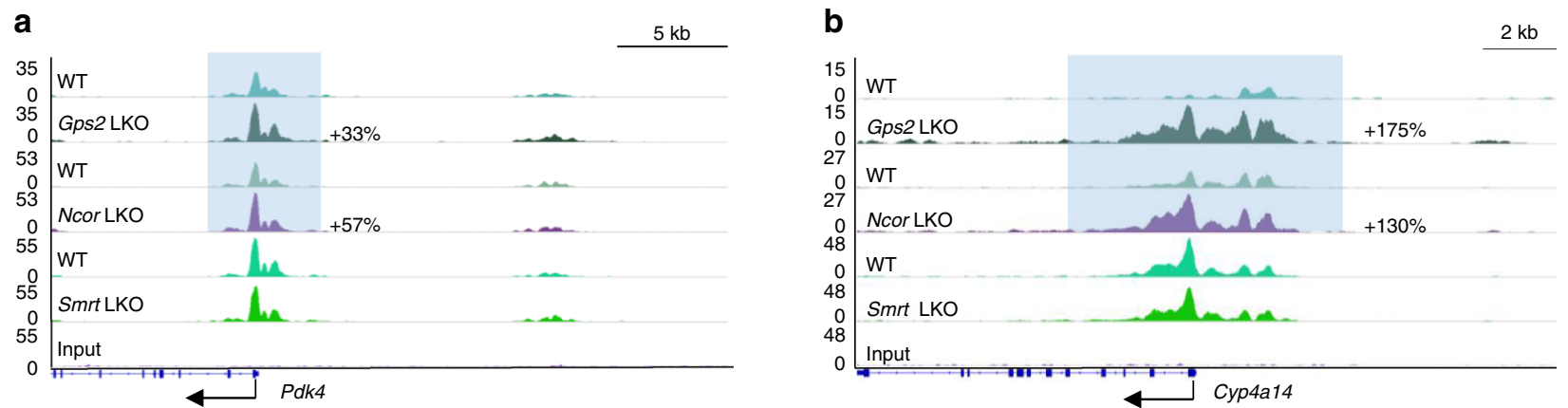

C

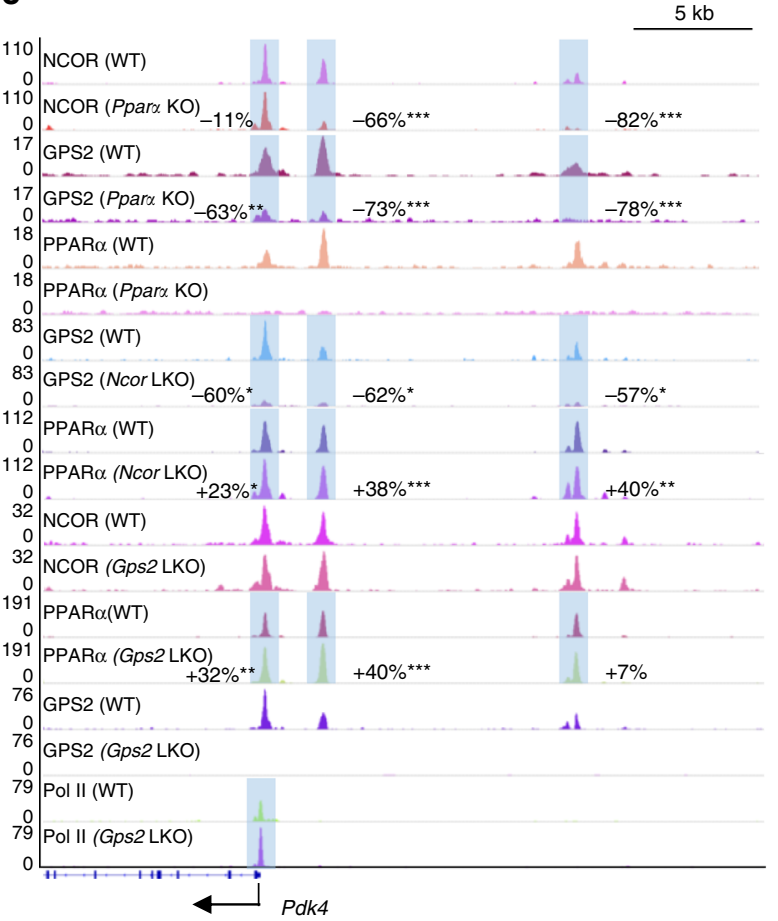

e

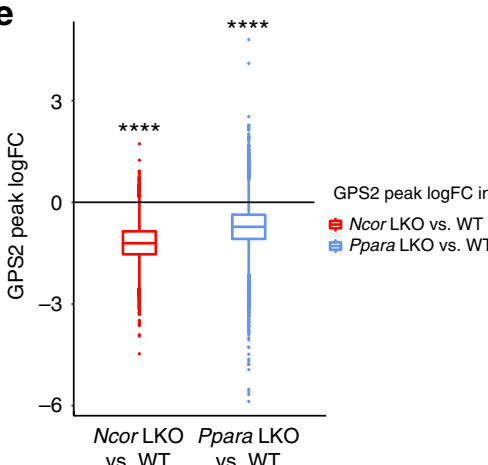

f

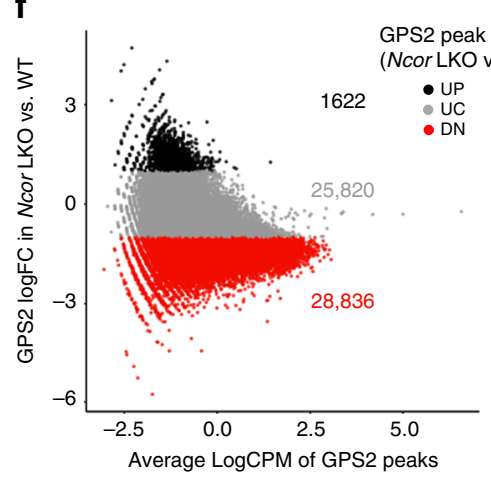

d

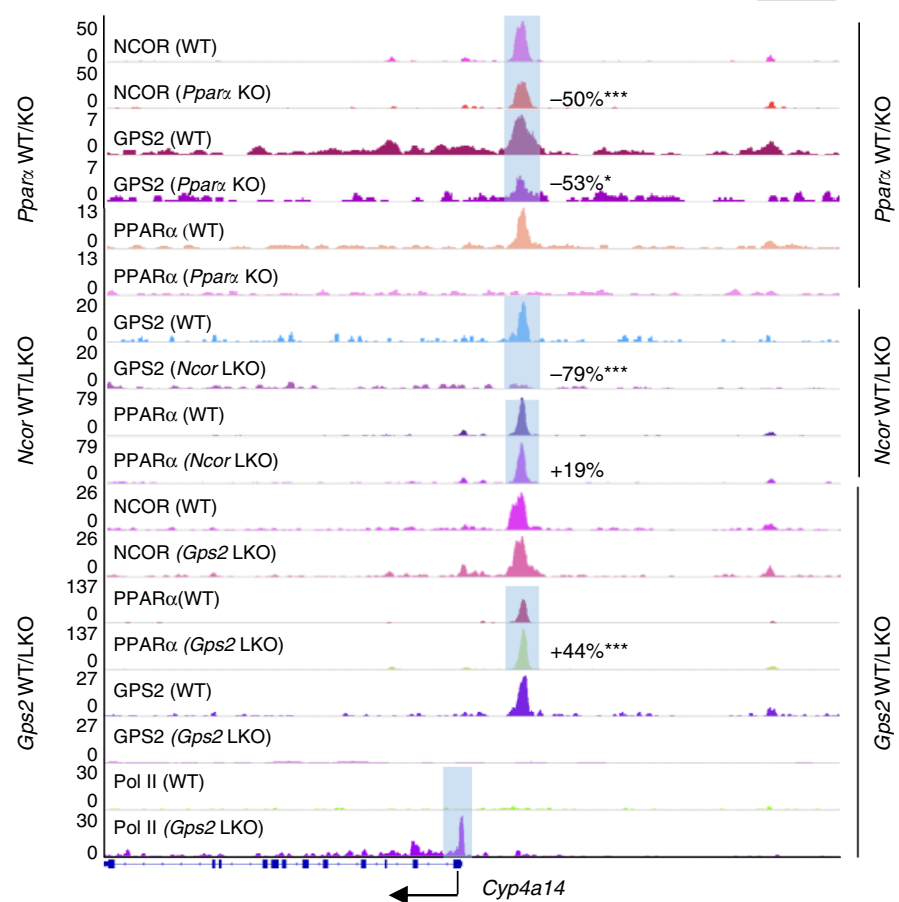

g

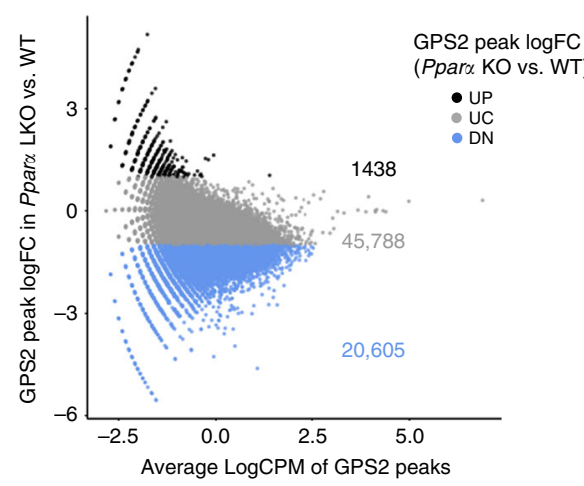

Fig. 5 GPS2 cooperates with NCOR to repress target gene expression. a, b ChIP-seq tracks representing H3K27ac at (a) Pdk4 and (b) Cyp4a14 loci in Gps2, Ncor and Smrt LKO and respective WT mouse livers. Percentage of increase represents logFC of each peak and is calculated from biological duplicates (for Gps2 LKO versus WT) or triplicates (for Ncor or Smrt LKO versus WT). c, d ChIP-seq tracks representing NCOR, GPS2, PPAR $\alpha$, and Pol II recruitment in Ppara KO, Ncor LKO, Gps2 LKO and respective WT livers at (c) Pdk4 and (d) Cyp4a14 loci. Percentage of increase represents logFC of each peak and is calculated from biological triplicates. e Boxplot representing average logFC of co-localized GPS2 peaks in Ncor LKO and Ppar $\alpha$ KO versus WT livers ( $n=3$ in each group). f, g MA plots showing the average logFC of GPS2 peaks between (f) Ncor LKO versus WT; and (g) Ppar $\alpha$ KO versus WT livers, against average $\log C P M$. UP, UN, and DN represents upregulated, unchanged, and downregulated peaks, respectively. All data are represented as mean \pm s.e.m. ${ }^{\star} P$ $<0.05,{ }^{\star \star} P<0.01,{ }^{\star \star \star} P<0.001$ 
LKO and in Ncor LKO livers, but not in Smrt LKO livers, at the Pdk4 and Cyp4a14 loci (Fig. 5a, b). Intriguingly, binding of GPS2 and NCOR was reduced in Ppara KO livers at the Pdk4 and Cyp4a14 loci (Fig. 5c, d, Supplementary Fig. 5g) while SMRT binding was not affected (Supplementary Fig. 5h). In addition, GPS2 recruitment to these loci relied on NCOR but not on SMRT and was abolished in Ncor LKO livers (Fig. 5c, d, Supplementary Fig. $5 \mathrm{~g}, \mathrm{i})$. The recruitment changes were not limited to above genes since global analysis of GPS2 ChIP-seq revealed reduced average binding in GPS2/NCOR or GPS2/PPARa co-localized loci in Ncor LKO or Ppara KO versus WT livers, respectively (Fig. 5e). Consistently, there were far more GPS2 peaks that were reduced comparing with the increased ones in Ncor LKO (Fig. 5f) or Ppara KO (Fig. 5g) versus WT livers. Similar analysis of PPAR $\alpha$ peaks in Gps2 and Ncor LKO versus WT liver showed increased average PPAR $\alpha$ recruitment (Supplementary Fig. 5j). The MA plot also revealed more enhanced PPARa binding in both Ncor and Gps2 LKO livers (Supplementary Fig. 5k, l). In addition, PPARa recruitment was slightly increased at NCORrepressed gene loci (i.e. upregulated in Ncor LKO versus WT mice), including the Pdk4 and Cyp4a14 loci (Fig. 5c, d, Supplementary Fig. 6a), but showed significant increase at GPS2repressed gene loci (Fig. 5c, d, Supplementary Fig. 6b). In contrast, NCOR, SMRT, and HDAC3 recruitment was only modestly changed in Gps2 LKO livers (Fig. 5c, d, Supplementary Fig. 6c).

The interactions of GPS2, NCOR, and PPARa were further investigated in vitro using co-immunoprecipitations. We found that full-length GPS2 enhanced the PPAR $\alpha$ interaction with NCOR (Supplementary Fig. 6d), while this was not seen with truncated GPS2 variants removing the NCOR-interaction domain (aa 61-94) or the PPARa-interaction domain (aa 100-155). These data support that the loss of GPS2 in hepatocytes de-stabilizes interactions of PPAR $a$ with the NCOR complex, and that release of the complex potentially facilitates the binding of RNA polymerase (Pol II) (Fig. 5c, d).

In sum, the above data suggest that GPS2 repression of PPAR $\alpha$ target genes in hepatocytes involves direct interactions of PPAR $\alpha$ with GPS2 and NCOR, which serves as the primary docking site of the corepressor complex to chromatin in hepatocytes.

Liver GPS2 expression correlates with NASH and fibrosis in humans. To determine the clinical relevance of GPS2 in human disease progression, we compared gene expression levels in both NASH (Fig. 6 and Supplementary Fig. 6e-j) and NAFLD (Fig. 6 and Supplementary Fig. 6e-j) human subjects from published transcriptome datasets (GSE83452 and GSE49541)15,16. The comparative analysis performed in a cohort of 44 control and 104 NASH liver samples identified 193 upregulated and 58 downregulated NASH signature genes as concluded in the previous publication (Fig. 6a). Expression of GPS2 was not different in different stages of NASH liver (Supplementary Fig. 6e). However, correlative analysis in the 104 NASH subjects showed a total of 74 genes significantly associated with GPS2 mRNA expression and 66 were positively correlated (Fig. 6b, Supplementary Table 2). Among them were fibrogenic genes including TGFB, TIMP1 (Supplementary Fig. 6f, g), and the inflammation marker CD68 (Supplementary Fig. 6h). Analysis of the other complex subunits revealed associations of these signature genes with NCOR, TBL1, TBLR1, and HDAC3, but notably not with SMRT (Fig. 6b). To further validate these results, we analyzed another transcriptome dataset (GSE49541) which contained 73 NAFLD patients at different fibrosis stages. GPS2 expression was positively correlated with the fibrogenic gene expression, including TGFB and TIMP1 (Fig. 6c, d).
Moreover, GPS2 expression was higher in NASH fibrosis compared to non-fibrosis liver biopsies (Fig. 6e) and was restored after weight loss in paired obese human subjects after dietary intervention or gastric bypass surgery (GABY) (Fig. 6f). The GPS2 mRNA was also positively correlated with HbAlc (Supplementary Fig. 6i). Intriguingly, correlative analysis of GPS2 and PPARA expression with the 72 GPS2-sensitive and NASH-related genes in the dataset from 104 human NASH patients showed an overall opposite correlation (Fig. 6g), while GPS2 and PPARA expression was not correlated (Supplementary Fig. 6j).

We conclude from above data that liver GPS2 expression and function could be causatively correlated with the progression of NAFLD towards NASH via regulating PPAR $\alpha$-coupled liver lipid metabolism (Fig. 6h).

\section{Discussion}

Our study uncovers a previously unknown role of GPS2 as an epigenetic modulator which inhibits liver lipid catabolism and thereby promotes the development of NALFD. Gps2 LKO caused the activation of promoters and enhancers controlling fatty acid oxidation genes in hepatocytes, which was dependent on PPARa activation. As a result of loss of PPARa repression, Gps2 LKO mice showed alleviated liver steatosis upon HFD feeding and improved fibrosis upon MCD feeding, due to increased lipid burning as detected by elevated ketone body levels. Remarkably, the protective phenotype of the Gps2 LKO mice is unique amongst hitherto described coregulator $\mathrm{KO}$ mouse models in the context of NAFLD as it is the only model which improved dietinduced fatty liver disease instead of worsening it (for references, see Introduction).

This hepatic function of GPS2 appears to be conserved between mice and humans as GPS2 levels correlate with fibrogenic and inflammatory gene expression in human NAFLD/NASH livers. Our study might thus provide hepatocyte-based epigenetic explanations for the diverse susceptibility in NAFLD/NASH patients to develop more severe stages of liver fibrosis and ultimately liver cancer, in addition to alterations in other cell types such as liver-resident immune cells ${ }^{1}$.

Previous studies in adipocytes ${ }^{20,42}$ and macrophages ${ }^{21}$ support a key role of GPS2 in repressing inflammation, and loss of GPS2 sensitized these cell-types to develop metabolic stressinduced inflammation (metaflammation) and insulin resistance, as shown for adipose tissue ${ }^{21}$. Importantly, this was not observed in the Gps2 LKO mice. Gene expression of representative proinflammatory genes was not significantly different between WT and LKO livers, and injection of LPS led to similar responses, as shown for $\mathrm{Ccl} 2$ and $\mathrm{Ccl} 7$, major GPS2 target genes in adipocytes and macrophages. These results are consistent with previous data demonstrating that in human hepatocytes GPS2 depletion had no effect on the inflammatory acute phase response but was essential for anti-inflammatory trans-repression of this response by nuclear receptors ${ }^{43}$.

Our earlier studies in human hepatocyte cell lines suggested a role of GPS2 in cholesterol metabolism which may influence the NAFLD phenotype ${ }^{38,44}$. However, chow diet-fed Gps2 LKO versus WT mice did not show differences in both serum and hepatic cholesterol levels despite triglyceride variation. Caution should be exercised in translating this aspect of the mouse phenotype to humans, as they differ in lipoprotein metabolism from mice due to absence of cholesterol ester transfer protein (CETP) ${ }^{45}$. There are also human-mouse differences in the regulation of cholesterol $7 a$-hydroxylase (CYP7A1), the rate-limiting enzyme in the classic bile acid synthetic pathway, by the nuclear receptors LXR, FXR, and SHP. While this pathway appears to be regulated by GPS2 in 
a

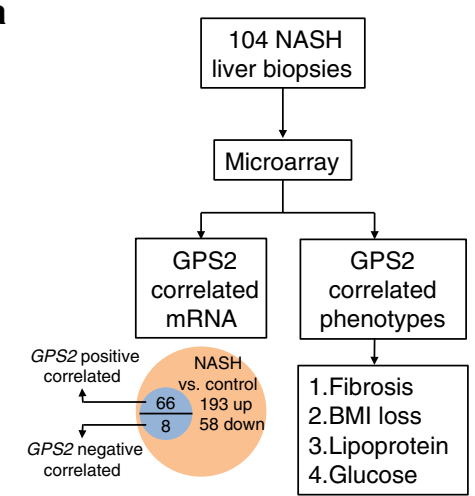

c

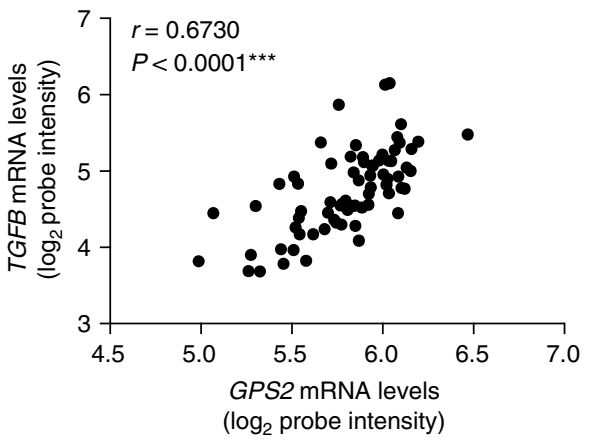

b

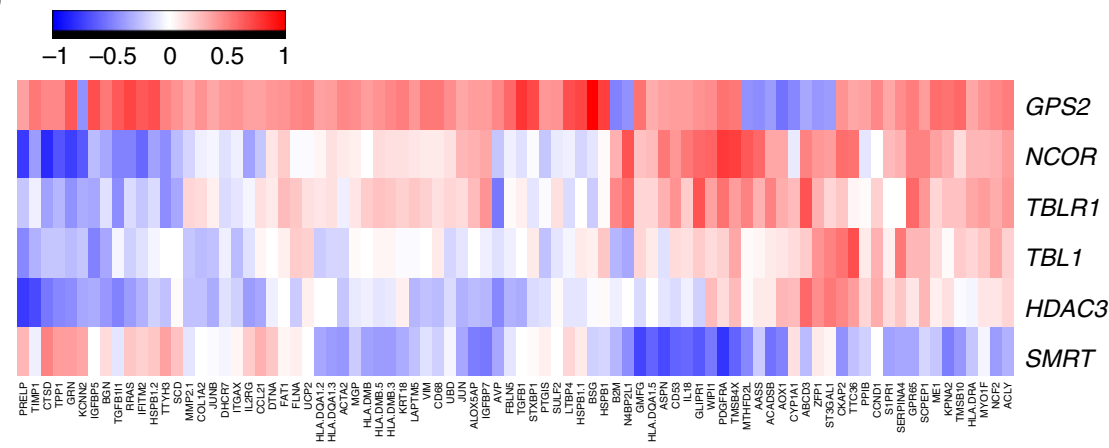

d

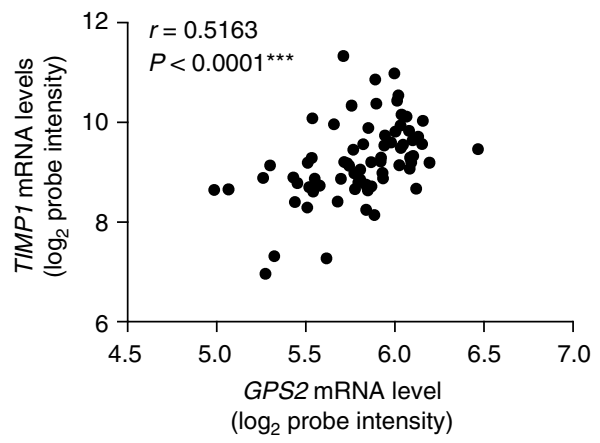

e

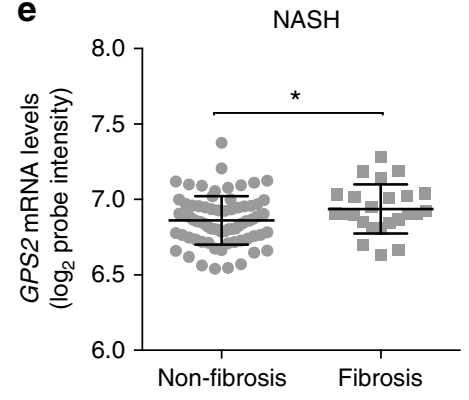

$\mathbf{f}$

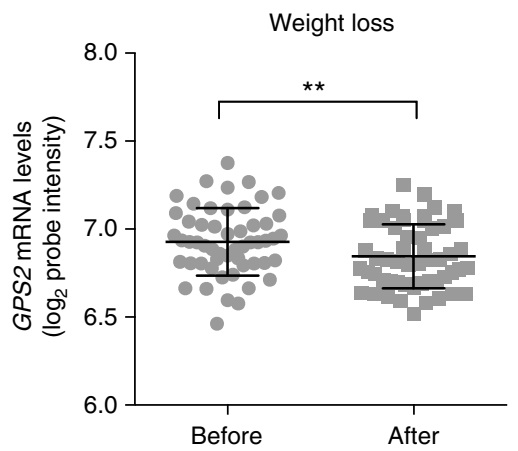

h

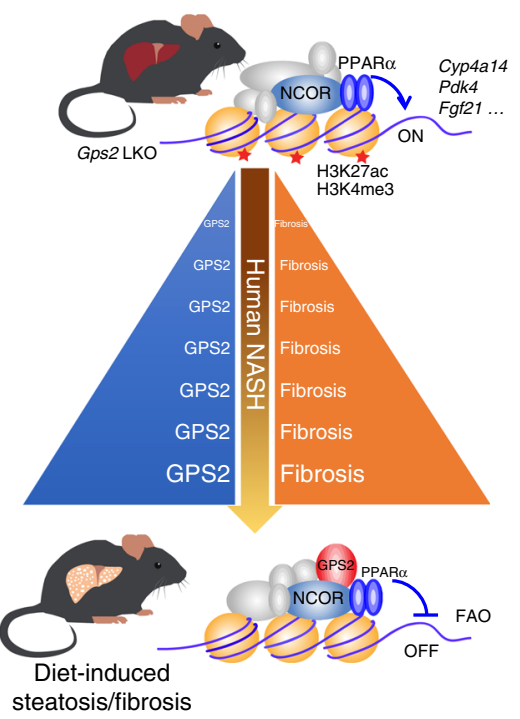

g

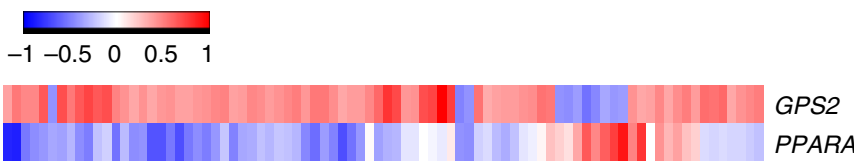

Fig. 6 GPS2 expression associates with NASH and fibrosis in human. a Flowchart representing the analysis of microarray results in the human liver biopsies. b Heatmap representing the rho correlation coefficient of GPS2 and other core subunits with NASH-regulated genes in human NASH liver biopsies. c, d Correlation analysis of GPS2 mRNA level with (c) TGFB and (d) TIMP1 in human NAFLD liver samples (GSE49541), $n=72$, non-parametric Spearman's test. e GPS2 mRNA level in non-fibrosis and fibrosis liver biopsies in the NASH subjects, $n$ (NASH non-fibrosis) $=74, n(N A S H$ fibrosis) $=24$, unpaired nonparametric Mann-Whitney test. f GPS2 mRNA level in NASH after weight loss in paired obese subjects, $n=54$, paired nonparametric Wilcoxon matched-pairs signed rank test. $\mathbf{g}$ Heatmap representing the rho correlation coefficient of GPS2 and PPARA with NASH-regulated genes in human NASH liver biospies $(n=104)$. $\mathbf{h}$ Model illustrating the causal relationship between GPS2 expression and PPAR $\alpha$ function in hepatocytes in the context of fatty liver disease. All data are represented as mean \pm s.e.m. ${ }^{\star} P<0.05,{ }^{\star \star} P<0.01,{ }^{\star \star \star} P<0.001$

human hepatocytes ${ }^{44}$, the activation of key LXR target genes by agonist treatment was similar in WT versus LKO mice, suggesting that liver LXR pathways in mice are not controlled by GPS2.

Despite the evidence that GPS2 likely functions as a core subunit of the NCOR/SMRT/HDAC3 corepressor complex in many metabolic tissues and cell types ${ }^{21,26,38,43,44}$, it may seem puzzling that the $\mathrm{KO}$ phenotypes for individual subunits were quite distinct with only partial overlap in specific pathways. We show here that in mouse hepatocytes GPS2 functionally cooperates with NCOR to repress PPARa-dependent pathways. This is in contrast to the situation in adipocytes and macrophages where GPS2 functionally cooperates with SMRT to repress proinflammatory pathways ${ }^{20,21}$. While the molecular reasons for this cell-type selective partnership remain enigmatic, they are not simply related to the differential expression and/or chromatinbinding features of NCOR and SMRT. Indeed, our liver cistrome 
analysis reveals that the core subunits GPS2, NCOR, SMRT, and HDAC3 co-occupy the majority of promoters and enhancers of GPS2-regulated genes, including PPARa-targets linked to fatty acid oxidation and to the LKO phenotype. This confirms our previous notion made in macrophages that the entire complex seems to be present at chromatin elements, yet this is not sufficient to predict function in the regulation of adjacent genes. These data collectively validate our recent concept of functionally distinct corepressor sub-complexes ${ }^{19}$.

Our new work identifies with GPS2 an additional core subunit of the complex that also interacts with PPARa and contributes to the overall repression in vivo. The Co-IP data and the inclusion of GPS2 mutants add in vitro support that GPS2 utilizes different domains to bind PPAR $\alpha$ vs. NCOR, and that this binding may be cooperative to increase PPARa interactions with NCOR. This is fully compatible with a model where cooperative interactions of two subunits explain why depletion of either NCOR or GPS2 reduces PPARa repression capacity and target gene expression in vivo.

There is evidence that GPS2 forms a stable core complex with NCOR/SMRT and TBL/TBLR1, supported by NMR structure data $^{23}$ and IP-mass spec data ${ }^{24,46}$. NCOR has been previously known to be the main receptor (also PPARa)-binding subunit of the complex, and our new data do not challenge this view. Indeed, our ChIP-seq data confirm that most GPS2 interactions with PPARa at chromatin are lost in Ncor KO hepatocytes, while NCOR interactions were not abolished in Gps2 KO hepatocytes. These results indicate that NCOR does not require GPS2 to interact with PPARa in vivo, thus contrasting the GPS2-NCOR bridging model suggested for agonist-bound PPAR $\gamma^{47}$.

However, there is so far no direct evidence whether this corepressor complex core forms stable ternary complexes with nuclear receptors, including PPARa. The current data rather suggest that repression in vivo results from dynamic transient interactions of the corepressor complex with TF targets ${ }^{22}$. NCOR and GPS2 are proposed to be largely intrinsically disordered in nature, which may reflect the involvement in many specific but relatively low-affinity interactions ${ }^{48}$.

Regarding the details of the PPARa interactions with GPS2 and NCOR, there is ample evidence suggesting that both proteins interact with distinct surfaces at the ligand-binding domain (LBD). In case of NCOR, the receptor interaction domain (RID) is largely unstructured but adopts upon interaction a helical peptide structure (the CORNR box) that interacts with the conserved cofactor surface at the LBD helices 3-5 (but excludes AF-2 helix 12), see for example, the structure of the PPAR $\alpha$ LBD with a corepressor peptide in the presence of an antagonist ${ }^{30,49,50}$. Although there is no structure data for any RID of GPS2, in vitro assays using receptor mutations and NCOR peptide competition strongly suggest that GPS2 binds to a surface that is different from the AF-2/NCOR-binding surface. In particular, the GPS2 surface seems exposed irrespective of the ligand status, marking a fundamental difference to the classic coactivator (LXXLL motif) corepressor (CORNR motif) surfaces ${ }^{38,47}$. One interesting in vivo scenario is that activation by endogenous PPARa agonists may lead to selective disruption of NCOR interactions, while GPS2 interactions may serve to stabilize the corepressor complex interactions, contributing to the GPS2 KO phenotype described here.

Remarkably, the functional cooperation of GPS2 with NCOR and its antagonism of PPARa is further supported by the correlation analysis of human NAFLD/NASH liver transcriptomes, suggesting conservation of the GPS2-NCOR partnership in driving NAFLD in mice and humans. The proposed link of GPS2 to NCOR in hepatocytes raises questions as to the different corresponding $\mathrm{KO}$ phenotypes. While Gps2 LKO resulted in improved liver steatosis and reduced lipid accumulation, Ncor LKO resulted in the opposite ${ }^{26,28}$. A likely explanation could be the partially distinct nuclear receptor and target gene selectivity of each subunit, with NCOR but not GPS2 repressing also LXRa in addition to PPARa ${ }^{28}$. As a consequence, lipogenic pathways were only up-regulated in Ncor LKO mice and likely contributed to the worsened fatty liver phenotype. Interestingly, the NCOR study provides some clues for how corepressor complex function, and in particular the subunit cooperation, might be further regulated to specify transcriptional outcomes. Nuclear receptor-selectivity seems regulated by insulin/AKT-mediated S1460 phosphorylation of NCOR, resulting in de-repression of LXR-dependent lipogenesis in the fed condition and PPAR $\alpha$-regulated fatty acid oxidation in the fasting condition [28].

The transcriptome comparison of Gps2 and Ncor LKO mice supported the different phenotypes, in particular the PPARaselective cooperation of NCOR with GPS2. Gene ontology analysis indicated that PPAR signaling was amongst the top pathways of the $30 \%$ of genes co-regulated by NCOR and GPS2, while LXR targets genes linked to lipogenesis were not co-regulated. Moreover, our analysis revealed interesting differences regarding the regulation of $F g f 21$, a major PPAR $\alpha$ target gene in liver. Gps 2 LKO resulted in an eight-fold increase in Fgf21 expression in the chow diet condition which was PPARa-dependent, the induction level almost equivalent to fasting effects, while in Ncor and Smrt LKO mice Fgf21 de-repression was not observed. The encoded FGF21 is a master regulator of metabolism which affects multiple pathways including fatty acid oxidation, and administration to mice has been shown to improve NAFLD ${ }^{13,51,52}$. The reason why $\mathrm{Fg} 21$ is selectively up-regulated in Gps2 but not in Ncor or Smrt LKO mice is unknown, but there is one report suggesting the HDAC3 subunit of the complex to be involved ${ }^{53}$. The study shows that pharmacological HDAC3 inhibition led to elevated Fgf21 expression in mouse and human liver cell lines. In addition to FGF21, most of the pathways and TFs (e.g. Rev-Erbs, Prox1) affected in Hdac3 LKO mice ${ }^{29,46}$ were not affected in Gps2 LKO mice, consistent with the broader target range of HDAC3 along with its intrinsic de-acetylation activity.

The unique phenotype of Gps2 LKO mice supports the idea that the GPS2 target range in vivo is limited, as compared to NCOR, SMRT, and HDAC 3 and to other coregulators expressed in the liver ${ }^{17}$. This implies that the manipulation of GPS2 function could be more selective than the manipulation of other coregulators. In conclusion, our study identifies GPS2 as an epigenome modifier and PPAR $\alpha$-selective corepressor in hepatocytes whose inhibition has the therapeutic potential to reverse the progression of NASH toward fibrosis.

\section{Methods}

Patients. The patient clinical information and human liver biopsy transcriptome data are from a previously published Belgium cohort ${ }^{15}$ collected from overweight individuals visiting the Obesity Clinic at the Antwerp University Hospital. The patient information and exclusion criteria were all described previously ${ }^{15}$. Briefly, the cohort used in this study is composed of 104 NASH patients with paired liver biopsies of 35 samples after dietary intervention and 39 samples after GABY (combined as weight loss group). The fibrosis stage was determined by pathological analysis of the liver biopsies. BMI (loss), HbAlc, HDL-c, and LDL-c were determined as described previously ${ }^{15}$, and the study was approved by the Ethical Committee of the Antwerp University Hospital (file 6/25/125).

\footnotetext{
Animals. Gps $2^{\text {flox/flox }}$ mice were generated at Ozgene Pty, Ltd. (Bentley DC, Australia) as previously described ${ }^{21}$. To generate LKO mice, Gps2 $2^{\text {flox/flox }}$ mice were crossed with Alb-Cre mice (B6.Cg-Speer6-ps1 1 (Al Alb-cre) $21 M g n / J$; Jackson Laboratory stock no. 003574). Gps $2^{\text {flox/flox }}$ mice from the same breedings were used as control (labeled as WT).

Ppar $\alpha$-deficient C57Bl/6J mice ${ }^{54}$ (B6;129S4-Ppar $\alpha^{\mathrm{tm} 1 \mathrm{Gonz}} / \mathrm{J}$; Stock no. 008154) were obtained from Jackson Laboratory.
} 
Liver-specific Gps2 and Ppara double KO mice were generated by breeding the

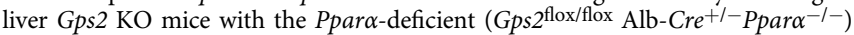
mice. Gps $2^{\text {flox/flox }} \mathrm{Alb}-\mathrm{Cr} e^{-l-}$ Ppar $\alpha^{-1-}$ mice were used as control.

Liver-specific Ncor and Smrt KO mice were generated as previously described ${ }^{26}$.

All animals were randomly assigned to each experimental group. The investigator was not blinded to the experimental groups during the study.

All animal experiments were approved by the respective national ethical boards (Swedish Board of Agriculture, Stockholm South, S28-12, S30-14, S135-12, S29-14, ID907) and conducted in accordance with the guidelines stated in the International Guiding Principles for Biomedical Research Involving Animals, developed by the Council for International Organizations of Medical Sciences (CIOMS). All mice strains were bred and maintained at the Center for Comparative Medicine at Karolinska Institutet and University Hospital (PKL, Huddinge, Sweden).

Cell culture studies. HEK293 (ATCC, CRL-1573) and AML12 (ATCC, CRL-2254) cells were tested for mycoplasma contamination. AML12 cells were authenticated by testing liver albumin expression before experiments. HEK293 were cultured in DMEM supplemented with $10 \%$ FBS and $1 \%$ antibiotics, and AML12 were cultured in DMEM/F12 high glucose supplemented with $10 \%$ FBS, $1 \%$ antibiotics, $1 \%$ ITS $(10 \mu \mathrm{g} / \mathrm{ml}$ insulin, $5.5 \mu \mathrm{g} / \mathrm{ml}$ transferrin, and $5 \mathrm{ng} / \mathrm{ml}$ selenium), and $40 \mathrm{ng} / \mathrm{ml}$ dexamethasone. Both cells were maintained at $37^{\circ} \mathrm{C}$ and $5 \% \mathrm{CO}_{2}$.

Seahorse analysis of OCR in AML12 cells. The Cell Mito Stress Test was performed according to the provided protocol. AML12 cells were seeded to the Seahorse XFe96 Cell Culture Microplate for $24 \mathrm{~h}$ before transduction with adenovirus shLuc (control) and shGPS2. After $36 \mathrm{~h}$, the cells were changed to Substrate-limited medium (Seahorse XF Base Medium plus 0.5 mM Glucose, 1.0 mM GlutaMAX, 0.5 $\mathrm{mM}$ Carnitine, and 1\% FBS, pH 7.4) and incubated for overnight. The cells were pre-incubated in Fatty Acid Oxidation Assay Buffer (KHB Buffer $(111 \mathrm{mM} \mathrm{NaCl}$, $4.7 \mathrm{mM} \mathrm{KCl}, 1.25 \mathrm{mM} \mathrm{CaCl}_{2}, 2.0 \mathrm{mM} \mathrm{MgSO}_{4}$, and $1.2 \mathrm{mM} \mathrm{NaH}_{2} \mathrm{PO}_{4}$ ) supplemented with $2.5 \mathrm{mM}$ glucose, $0.5 \mathrm{mM}$ carnitine, and $5 \mathrm{mM}$ HEPES, pH 7.4) at $37^{\circ}$ $\mathrm{C}$ for $45 \mathrm{~min}$ in a non- $\mathrm{CO}_{2}$ incubator. BSA (control) or BSA-palmitate were added just prior to starting the assay. After subsequent injecting and mixing of the compounds (assay concentrations: $2.0 \mu \mathrm{M}$ oligomycin, $2.0 \mu \mathrm{M}$ FCCP, and $0.5 \mu \mathrm{M}$ Rotenone/antimycin A), the OCR was determined in all wells three times. The results were normalized with the protein quantity of each corresponding well.

Metabolic studies in mice. 7-8-week-old WT and LKO mice were fed with a $60 \%$ fat diet (HFD, Research Diets, D12492) or with a $10 \%$ fat diet (LFD, Research Diets, D12450) for 12 weeks. To establish a fibrosis model, mice were fed with a MCD (Research Diets, A02082002B) or with a control diet (Research Diets, A02082003B) for 4 weeks. During the experiments, body weight was assessed at different time points. OGTT and ITT were performed in the chow-diet and HFD feeding mice as previously described ${ }^{21}$. Corresponding blood glucose levels were measured at the indicated time points using a glucometer (Accu-Chek Performa, Roche). Plasma insulin (Mercodia, 10-1247-01), FGF21 (R\&D Systems, DF2100), $\beta$-Hydroxybutyrate (Cayman, 700190) and hydroxyproline levels (Sigma,

MAK008) were determined by ELISA.

Liver TG and cholesterol were extracted and measured with a colorimetric diagnostic kit (WAKO diagnostics) as previously described ${ }^{21}$. Plasma lipoproteins were fractionated from $2.5 \mu \mathrm{l}$ of each sample using a Superose 6 PC 3.2/30 column (GE Healthcare) followed by online determination of TGs and cholesterols as previously described ${ }^{21}$. The lipid concentrations of the different lipoprotein fractions were calculated after integration of individual chromatograms.

Lipase activity is measured using the assay kit (Sigma Aldrich, MAK046) according to manufacturer instructions. In brief, $30 \mathrm{~min}$ after heparin injection, the mice were sacrificed and mouse plasma was separated by centrifuging for $10 \mathrm{~min}$, 3000 r.p.m. at $4{ }^{\circ} \mathrm{C}$. Plasma was used to test the lipase activity with (HL activity) or without $1 \mathrm{M} \mathrm{NaCl}$, LPL was determined by substracting $\mathrm{HL}$ activity from the total lipase activity.

Morphometric analyses. The tissue processing and staining were performed in morphological phenotype analysis (FENO) core facility at Department of Laboratory Medicine, Karolinska Institute. Briefly, the mice liver tissue samples were fixed in $3 \%$ formaldehyde solution overnight and embedded in paraffin. Tissue slides were stained with $\mathrm{H} \& \mathrm{E}$ for the evaluation of the tissue morphology, with Sirius red for collagen-specific staining, or with F4/80 (Abcam, ab6649) for liver resident macrophages, following standardized protocols. Sirius red and F4/80 positive areas were quantified using imageJ.

All mice used in the studies were male, between 7 and 16 weeks old at the time of the experiment starting point, and randomized before any experiment was started. All animal experiments were approved by the respective national ethical boards (Swedish Board of Agriculture) and conducted in accordance with the guidelines stated in the International Guiding Principles for Biomedical Research Involving Animals, developed by the CIOMS. All mice strains were bred and maintained at the Center for Comparative Medicine at Karolinska Institutet and University Hospital (PKL, Huddinge, Sweden).
Microarray analysis. Raw-intensity expression files (.CEL files) (GSE83452; GSE48452; GSE73299; GSE49388; GSE54192) were imported to R and Bioconductor using the Oligo package ${ }^{55}$. The same package was used for quantile normalization, background correction, and summarization by robust multichip average preprocessing (RMA). The normalized log2-transformed expression values were then imported to the Limma ${ }^{56}$ package for differential-expression analysis by linear modeling. A paired design was used to remove the batch effect between the biological replicates. Furthermore, genes with low expression (less than the 95th quantile of the negative-control probes) were removed. Genes with a $P$-value of $<0.05$, after adjusting for multiple hypothesis testing using the FDR method, were defined as being differentially expressed.

Correlation matrix was calculated based on expression values in log2 scale of 251 candidate NASH genes ${ }^{15}$ and GPS2-NCOR-HDAC3 complex components from 104 NASH livers at baseline. Genes whose expression significantly correlated with GPS2 expression were selected and their correlation with GPS2-NCOR-HDAC3 complex components was plotted as heatmap.

qPCR analysis. Total RNA was extracted from snap-frozen liver tissues using Trizol Reagent (Thermo Fisher Scientific, 15596-026) and RNeasy RNA Mini Kit (Qiagen, 74104). Complementary DNAs were synthesized using M-MLV Reverse Transcriptase (Life Technologies, 28025-021). QPCR was performed using the ABI Prism 7500 PCR system (Applied Biosystems). 36b4 (gene encoding acidic ribosomal phosphoprotein P0, also called Rplp0) were used for normalization to quantify relative mRNA expression levels. Relative changes in mRNA expression were calculated using the comparative cycle method $\left(2^{-\Delta \Delta C t}\right)$. Primers are listed in Supplementary Table 1.

RNA-seq. RNA was extracted from mice liver biopsies as described above. RNA quality was assessed by 2200 TapeStation Instrument (Agilent). PolyA RNA selection was performed using the Illumina TruSeq RNA Sample Preparation Kit according to the manufacturer's protocol. RNA-seq libraries were prepared and sequenced on the Illumina HiSeq 2000 platform at Bioinformatics and Expression Analysis core facility (BEA, Karolinska Institutet, Sweden). Preprocessed reads were aligned to the $\mathrm{mm} 9$ transcriptome using the HISAT2 program, and Hypergeometric Optimization of Motif EnRichment (HOMER, http://homer.salk.edu/ homer) was used to create the tag directory and count tags in all exons. Raw tag counts were imported in to $\mathrm{R}$ and Bioconductor and edgeR package was used to determine differential gene expression.

ChIP and ChIP-seq sample preparation. ChIP samples were prepared as described previously ${ }^{21}$, with minor modifications. Briefly, fresh livers were chopped into small pieces and crosslinked with $1 \%$ formaldehyde (ThermoFisher, 28906) in PBS for 10 min for histone modifications, or double crosslinked with $2 \mathrm{mM}$ disuccinimidyl glutarate (DSG) for $30 \mathrm{~min}$, followed by $1 \%$ formaldehyde for $10 \mathrm{~min}$, for TFs and GPS2. The reaction was stopped with glycine at a final concentration of $0.125 \mathrm{M}$ for $5 \mathrm{~min}$.

Liver pieces were disaggregated in ice-cold PBS with protease inhibotor using Dounce Homogenizer first with loose and later with tight pestle (Fisher Science, FB56691). Nuclei were isolated using lysis buffer 1 (50 mM Hepes-KOH, pH 7.5, $140 \mathrm{mM} \mathrm{NaCl}, 1 \mathrm{mM}$ EDTA, 10\% glycerol, 0.5\% IGEPAL CA-630, and $0.25 \%$ Triton X-100), lysis buffer 2 (10 mM Tris-HCl, pH 8.0, $200 \mathrm{mM} \mathrm{NaCl}, 1 \mathrm{mM}$ EDTA, and 0.5 mM EGTA), and lysis buffer $3(10 \mathrm{mM}$ Tris- $\mathrm{HCl}, \mathrm{pH} 8.0,100 \mathrm{mM}$ $\mathrm{NaCl}, 1 \mathrm{mM}$ EDTA, $0.5 \mathrm{mM}$ EGTA, $0.1 \% \mathrm{Na}$-deoxycholate, and $0.5 \% \mathrm{~N}$ Lauroylsarcosine), and subsequently sonicated for $30 \mathrm{~min}$ ( $30 \mathrm{~s} \mathrm{ON} / 30 \mathrm{~s}$ OFF) in the Bioruptor Pico (Diagenode). Protein A Dynabeads (Invitrogen) were incubated $\mathrm{O} / \mathrm{N}$ with the antibodies. Each lysate was immunoprecipitated with the following antibodies: control rabbit IgG (Santa Cruz, sc-2027, 1-5 $\mu \mathrm{g}$ ), anti-H3K4me3 (Abcam, ab8580, $1 \mu \mathrm{g}$ ), anti-H3K27ac (Abcam, ab4729, $1 \mu \mathrm{g}$ ), anti-PPARa (Millipore MAB3890, $5 \mu \mathrm{g}$ ), anti-Polymerase II (Biolegend, 664906, $5 \mu \mathrm{g}$ ), antiNCOR (Bethyl laboratories, A301-145A, $4 \mu \mathrm{g}$ ), anti-SMRT (Bethyl laboratories, A301-147A, $4 \mu \mathrm{g}$ ), anti-HDAC3 (Santa Cruz, sc-11417, $5 \mu \mathrm{g}$ ), and anti-GPS2 (4 $\mu \mathrm{g})$. Formaldehyde cross-linking was reversed overnight at $65^{\circ} \mathrm{C}$, and the immunoprecipitated DNA was purified using the QIAquick PCR purification kit (Qiagen). Primers for the ChIP qPCR are listed in Supplementary Table 1.

To prepare the ChIP-seq samples, the same ChIP protocol was followed, but using the ChIP DNA Clean and Concentrator Capped Zymo-Spin I (Zymo Research) purification kit. Two to four ChIPs were pooled during the final step of the purification to obtain concentrated material.

For library preparation and sequencing, 2-10 ng of ChIPed DNA was processed using Rubicon ThruPLEX DNA-seq kit (TAKARA) or processed at the EMBL Genomics Core Facility (Heidelberg, Germany) using standard protocols, and sequenced in the Illumina HiSeq 2000 (50SE reads, EMBL) or NextSeq 550 (75SE reads, BEA Core Facility, Karolinska Institutet, Sweden).

ChIP-seq data analysis. The computations were performed on resources provided by SNIC through Uppsala Multidisciplinary Center for Advanced Computational Science (UPPMAX) under Project SNIC 2018/8-122. Analysis was performed as previously described ${ }^{21}$. Sequencing files (fastq files), provided by the EMBL Genomics (Heidelberg, Germany) or BEA (Karolinska Institutet, Sweden) Core 
Facility, and raw data from the published ChIP-seq data (PPARa: GSE61817; H3K4me1: GSE29184; NCOR: GSE26345; SMRT: GSE51045) were aligned to the NCBI37/mm9 version of the mouse reference genome, using Bowtie ${ }^{57}$. Peaks were identified using HOMER package ${ }^{58}$.

Peak heights were normalized to the total number of uniquely mapped reads and displayed in integrative genomics viewer $(\mathrm{IGV})^{59}$ as the number of tags per 10 million tags. The sequences found in GPS2 peaks were subjected to motif analysis to identify potentially over-represented TF-binding sites using HOMER . Heat-map clustering of the peaks was performed in Cluster $3.0^{60}$ using selforganizing maps, and then visualized in TreeView ${ }^{61}$. For statistical analysis of the peaks, raw tag counts were imported into $\mathrm{R}$ and Bioconductor and edgeR package was used to identify potential differential-binding sites ${ }^{62,63}$.

Western blot analysis. Samples were lysed in RIPA buffer supplemented with protease and phosphatase inhibitors and were diluted to a concentration of $20 \mu \mathrm{g}$ of protein and heated at $98^{\circ} \mathrm{C}$ for $10 \mathrm{~min}$. Proteins were separated by SDS-PAGE electrophoresis and transferred to PVDF membranes (Amersham International). Blocking reagent (SuperBlock ${ }^{\mathrm{mI}} \mathrm{T}$ T20 (PBS) blocking buffer, Thermo Fisher Scientific) were incubated for $1 \mathrm{~h}$, and primary antibody was incubated overnight at $4{ }^{\circ} \mathrm{C}$ in the blocking solution. The antibodies and their concentrations are the following: anti-GPS2 $2^{38,43}$ (generated from Agrisera; $1: 3000$ ), anti- $\beta$-actin (Abcam, ab8226; 1 : 30,000), anti-HDAC3 (Santa Cruz, sc11417; 1:3000), anti-PPARa (Cayman, 101710; 1:3000), anti-HA (BioLegend, 901514; 1:5000), and anti-Flag (SigmaAldrich, F7425; 1:5000). After several washes in PBST (PBS with 0.05\% Tween 20), horseradish peroxidase (HRP)-labeled secondary antibodies (1:5000) were incubated for $1 \mathrm{~h}$ at room temperature in PBST. Membranes were developed with ECL western-blotting substrate (BioRad, 1705061).

Co-immunoprecipitations. HEK293 cells were co-transfected with (1) WT or truncated pcDNA3-HA-GPS2 and expression plasmids for Flag-tagged PPARa to mapping the interaction domain of GPS2 and PPAR $\alpha$ or (2) WT or truncated HAGPS2, HA-PPARa, and Flag-NCOR to determine the affinity of NCOR and PPARa in the absence and the presence of WT or truncated GPS2. Cells were lysed $48 \mathrm{~h}$ after transfection, and the lysate was incubated with anti-Flag (Sigma-Aldrich, F7425) coupled to protein A magnetic beads for $3 \mathrm{~h}$, at $4^{\circ} \mathrm{C}(15 \mu \mathrm{l}$ beads were preincubated with $2 \mu \mathrm{g}$ of antibody for $2 \mathrm{~h}$, at $4^{\circ} \mathrm{C}$ ). Beads were washed with lysis buffer five times and eluted at $98^{\circ} \mathrm{C}$ for $10 \mathrm{~min}$. The eluted sample was loaded in an acrylamide gel by following the western blot protocol, and blotted with anti-Flag or anti-HA. Whole-cell lysis was used as input.

Statistical analysis. All the replicate experiments (including cell and mouse-based experiments) are biological replicates, which were repeated at least two times in the lab. Sample size was not pre-specified statistically. D'Agostino and Pearson normality test was used to determine the normal distribution. The variance within each group of data was compared using $F$-test (two groups) or Brown-Forsythe test (more than two groups). All statistical tests were performed using GraphPad Prism 6.0b (GraphPad Software, Inc., La Jolla, CA), and all data are represented as mean \pm s.e.m. Statistical tests were assessed after confirming that the data met appropriate assumptions (normality, homogenous variance, and independent sampling). All statistical tests were two-tailed, and $p<0.05$ was defined as significant. No statistical methods were used to predetermine sample size. No samples or animals were excluded from the analysis.

Reporting summary. Further information on experimental design is available in the Nature Research Reporting Summary linked to this article.

\section{Data availability}

Gene expression RNA-seq data and ChIP-seq data have been deposited at the NCBI Gene Expression Omnibus (GEO) accession numbers are GSE113157. The authors declare that all data supporting the findings of this study are available in figshare with https://doi.org/10.6084/m9.figshare.7637504. Other data are available from the corresponding author upon request.

Received: 2 May 2018 Accepted: 12 March 2019

Published online: 11 April 2019

\section{References}

1. Musso, G., Cassader, M. \& Gambino, R. Non-alcoholic steatohepatitis: emerging molecular targets and therapeutic strategies. Nat. Rev. Drug Discov. 15, 249-274 (2016).

2. Eslam, M., Valenti, L. \& Romeo, S. Genetics and epigenetics of NAFLD and NASH: clinical impact. J. Hepatol. 68, 268-279 (2018).

3. Hardy, T. \& Mann, D. A. Epigenetics in liver disease: from biology to therapeutics. Gut 65, 1895-1905 (2016)
4. Arab, J. P., Karpen, S. J., Dawson, P. A., Arrese, M. \& Trauner, M. Bile acids and nonalcoholic fatty liver disease: molecular insights and therapeutic perspectives. Hepatology 65, 350-362 (2017).

5. Tanaka, N., Aoyama, T., Kimura, S. \& Gonzalez, F. J. Targeting nuclear receptors for the treatment of fatty liver disease. Pharmacol. Ther. 179, 142-157 (2017).

6. Jakobsson, T., Vedin, L. L. \& Parini, P. Potential role of thyroid receptor beta agonists in the treatment of hyperlipidemia. Drugs 77, 1613-1621 (2017).

7. Pawlak, M., Lefebvre, P. \& Staels, B. Molecular mechanism of PPARalpha action and its impact on lipid metabolism, inflammation and fibrosis in nonalcoholic fatty liver disease. J. Hepatol. 62, 720-733 (2015).

8. Monetti, M. et al. Dissociation of hepatic steatosis and insulin resistance in mice overexpressing DGAT in the liver. Cell. Metab. 6, 69-78 (2007).

9. Goedeke, L. et al. Acetyl-CoA carboxylase inhibition reverses NAFLD and hepatic insulin resistance but promotes hypertriglyceridemia in rodents. Hepatology 68, 2197-2211 (2018).

10. Fabbrini, E., Sullivan, S. \& Klein, S. Obesity and nonalcoholic fatty liver disease: biochemical, metabolic, and clinical implications. Hepatology $\mathbf{5 1}$ 679-689 (2010).

11. Li, Y. et al. AMPK phosphorylates and inhibits SREBP activity to attenuate hepatic steatosis and atherosclerosis in diet-induced insulin-resistant mice. Cell. Metab. 13, 376-388 (2011).

12. Ding, X., Saxena, N. K., Lin, S., Gupta, N. A. \& Anania, F. A. Exendin-4, a glucagon-like protein-1 (GLP-1) receptor agonist, reverses hepatic steatosis in ob/ob mice. Hepatology 43, 173-181 (2006).

13. $\mathrm{Xu}, \mathrm{J}$. et al. Fibroblast growth factor 21 reverses hepatic steatosis, increases energy expenditure, and improves insulin sensitivity in diet-induced obese mice. Diabetes 58, 250-259 (2009).

14. Alvarez-Sola, G. et al. Fibroblast growth factor 15/19 (FGF15/19) protects from diet-induced hepatic steatosis: development of an FGF19-based chimeric molecule to promote fatty liver regeneration. Gut 66, 1818-1828 (2017).

15. Lefebvre, P. et al. Interspecies NASH disease activity whole-genome profiling identifies a fibrogenic role of PPARalpha-regulated dermatopontin. JCI Insight 2, e92264 (2017).

16. Murphy, S. K. et al. Relationship between methylome and transcriptome in patients with nonalcoholic fatty liver disease. Gastroenterology 145, 1076-1087 (2013).

17. Sommerfeld, A., Krones-Herzig, A. \& Herzig, S. Transcriptional co-factors and hepatic energy metabolism. Mol. Cell. Endocrinol. 332, 21-31 (2011).

18. Giudici, M., Goni, S., Fan, R. \& Treuter, E. Nuclear receptor coregulators in metabolism and disease. Handb. Exp. Pharmacol. 233, 95-135 (2016).

19. Treuter, E., Fan, R., Huang, Z., Jakobsson, T. \& Venteclef, N. Transcriptional repression in macrophages-basic mechanisms and alterations in metabolic inflammatory diseases. FEBS Lett. 591, 2959-2977 (2017).

20. Toubal, A. et al. SMRT-GPS2 corepressor pathway dysregulation coincides with obesity-linked adipocyte inflammation. J. Clin. Invest. 123, 362-379 (2013).

21. Fan, R. et al. Loss of the co-repressor GPS2 sensitizes macrophage activation upon metabolic stress induced by obesity and type 2 diabetes. Nat. Med. 22, 780-791 (2016).

22. Emmett, M. J. \& Lazar, M. A. Integrative regulation of physiology by histone deacetylase 3. Nat. Rev. Mol. Cell Biol. 20, 102-115 (2018).

23. Oberoi, J. et al. Structural basis for the assembly of the SMRT/NCoR core transcriptional repression machinery. Nat. Struct. Mol. Biol. 18, 177-184 (2011).

24. Zhang, J., Kalkum, M., Chait, B. T. \& Roeder, R. G. The N-CoR-HDAC3 nuclear receptor corepressor complex inhibits the JNK pathway through the integral subunit GPS2. Mol. Cell 9, 611-623 (2002).

25. Pourcet, B., Pineda-Torra, I., Derudas, B., Staels, B. \& Glineur, C. SUMOylation of human peroxisome proliferator-activated receptor alpha inhibits its trans-activity through the recruitment of the nuclear corepressor NCoR. J. Biol. Chem. 285, 5983-5992 (2010).

26. Shimizu, H. et al. NCoR1 and SMRT play unique roles in thyroid hormone action in vivo. Mol. Cell. Biol. 35, 555-565 (2015).

27. Jeyakumar, M., Liu, X. F., Erdjument-Bromage, H., Tempst, P. \& Bagchi, M. K. Phosphorylation of thyroid hormone receptor-associated nuclear receptor corepressor holocomplex by the DNA-dependent protein kinase enhances its histone deacetylase activity. J. Biol. Chem. 282, 9312-9322 (2007).

28. Jo, Y. S. et al. Phosphorylation of the nuclear receptor corepressor 1 by protein kinase B switches its corepressor targets in the liver in mice. Hepatology $\mathbf{6 2}$, 1606-1618 (2015).

29. Feng, D. et al. A circadian rhythm orchestrated by histone deacetylase 3 controls hepatic lipid metabolism. Science 331, 1315-1319 (2011).

30. Dowell, P. et al. Identification of nuclear receptor corepressor as a peroxisome proliferator-activated receptor alpha interacting protein. J. Biol. Chem. 274, 15901-15907 (1999). 
31. Yu, C. et al. The nuclear receptor corepressors NCoR and SMRT decrease peroxisome proliferator-activated receptor gamma transcriptional activity and repress 3T3-L1 adipogenesis. J. Biol. Chem. 280, 13600-13605 (2005).

32. Guan, D. et al. Diet-induced circadian enhancer remodeling synchronizes opposing hepatic lipid metabolic processes. Cell 174, 831-842 e812 (2018).

33. Kulozik, P. et al. Hepatic deficiency in transcriptional cofactor TBL1 promotes liver steatosis and hypertriglyceridemia. Cell. Metab. 13, 389-400 (2011).

34. Robinson, M. W., Harmon, C. \& O'Farrelly, C. Liver immunology and its role in inflammation and homeostasis. Cell. Mol. Immunol. 13, 267-276 (2016).

35. Lefebvre, P., Chinetti, G., Fruchart, J. C. \& Staels, B. Sorting out the roles of PPAR alpha in energy metabolism and vascular homeostasis. J. Clin. Invest. 116, 571-580 (2006)

36. Montagner, A. et al. Liver PPARalpha is crucial for whole-body fatty acid homeostasis and is protective against NAFLD. Gut 65, 1202-1214 (2016).

37. Kersten, S. Integrated physiology and systems biology of PPARalpha. Mol. Metab. 3, 354-371 (2014).

38. Jakobsson, T. et al. GPS2 is required for cholesterol efflux by triggering histone demethylation, LXR recruitment, and coregulator assembly at the ABCG1 locus. Mol. Cell 34, 510-518 (2009).

39. Shen, Y. et al. A map of the cis-regulatory sequences in the mouse genome Nature 488, 116-120 (2012).

40. Lee, J. M. et al. Nutrient-sensing nuclear receptors coordinate autophagy. Nature 516, 112-115 (2014).

41. Sun, Z. et al. Deacetylase-independent function of HDAC3 in transcription and metabolism requires nuclear receptor corepressor. Mol. Cell 52, 769-782 (2013).

42. Cardamone, M. D. et al. A protective strategy against hyperinflammatory responses requiring the nontranscriptional actions of GPS2. Mol. Cell 46, 91-104 (2012).

43. Venteclef, N. et al. GPS2-dependent corepressor/SUMO pathways govern anti-inflammatory actions of LRH-1 and LXRbeta in the hepatic acute phase response. Genes Dev. 24, 381-395 (2010).

44. Sanyal, S. et al. Involvement of corepressor complex subunit GPS2 in transcriptional pathways governing human bile acid biosynthesis. Proc. Natl Acad. Sci. USA 104, 15665-15670 (2007).

45. Grass, D. S. et al. Transgenic mice expressing both human apolipoprotein B and human CETP have a lipoprotein cholesterol distribution similar to that of normolipidemic humans. J. Lipid Res. 36, 1082-1091 (1995).

46. Armour, S. M. et al. An HDAC3-PROX1 corepressor module acts on HNF4alpha to control hepatic triglycerides. Nat. Commun. 8, 549 (2017).

47. Guo, C. et al. The optimal corepressor function of nuclear receptor corepressor (NCoR) for peroxisome proliferator-activated receptor gamma requires $\mathrm{G}$ protein pathway suppressor 2. J. Biol. Chem. 290, 3666-3679 (2015).

48. Watson, P. J., Fairall, L. \& Schwabe, J. W. Nuclear hormone receptor corepressors: structure and function. Mol. Cell. Endocrinol. 348, 440-449 (2012).

49. Xu, H. E. et al. Structural basis for antagonist-mediated recruitment of nuclear co-repressors by PPARalpha. Nature 415, 813-817 (2002).

50. Liu, M. H. et al. A natural polymorphism in peroxisome proliferator-activated receptor-alpha hinge region attenuates transcription due to defective release of nuclear receptor corepressor from chromatin. Mol. Endocrinol. 22, 1078-1092 (2008).

51. Rakhshandehroo, M., Knoch, B., Muller, M. \& Kersten, S. Peroxisome proliferator-activated receptor alpha target genes. PPAR Res. 61, 393-416 (2010).

52. Badman, M. K. et al. Hepatic fibroblast growth factor 21 is regulated by PPARalpha and is a key mediator of hepatic lipid metabolism in ketotic states. Cell. Metab. 5, 426-437 (2007).

53. Li, H. et al. Sodium butyrate stimulates expression of fibroblast growth factor 21 in liver by inhibition of histone deacetylase 3. Diabetes 61, 797-806 (2012).

54. Lee, S. S. et al. Targeted disruption of the alpha isoform of the peroxisome proliferator-activated receptor gene in mice results in abolishment of the pleiotropic effects of peroxisome proliferators. Mol. Cell. Biol. 15, 3012-3022 (1995).

55. Carvalho, B. S. \& Irizarry, R. A. A framework for oligonucleotide microarray preprocessing. Bioinformatics 26, 2363-2367 (2010).

56. Ritchie, M. E. et al. limma powers differential expression analyses for RNAsequencing and microarray studies. Nucleic Acids Res. 43, e47 (2015)

57. Langmead, B., Trapnell, C., Pop, M. \& Salzberg, S. L. Ultrafast and memoryefficient alignment of short DNA sequences to the human genome. Genome Biol. 10, R25 (2009).

58. Heinz, S. et al. Simple combinations of lineage-determining transcription factors prime cis-regulatory elements required for macrophage and B cell identities. Mol. Cell 38, 576-589 (2010).

59. Robinson, J. T. et al. Integrative genomics viewer. Nat. Biotechnol. 29, 24-26 (2011).

60. de Hoon, M. J., Imoto, S., Nolan, J. \& Miyano, S. Open source clustering software. Bioinformatics 20, 1453-1454 (2004).
61. Page, R. D. TreeView: an application to display phylogenetic trees on personal computers. Comput. Appl. Biosci. 12, 357-358 (1996).

62. Robinson, M. D., McCarthy, D. J. \& Smyth, G. K. edgeR: a Bioconductor package for differential expression analysis of digital gene expression data. Bioinformatics 26, 139-140 (2010)

63. McCarthy, D. J., Chen, Y. \& Smyth, G. K. Differential expression analysis of multifactor RNA-Seq experiments with respect to biological variation. Nucleic Acids Res. 40, 4288-4297 (2012).

\section{Acknowledgements}

We acknowledge Ozgene Pty, Ltd. for generating floxed GPS2 mice. We acknowledge V Benes and the team at the EMBL Genomics Core Facility and F. Fagerström-Billai and co-workers at the BEA Core Facility (Karolinska Institutet) for ChIP-seq library preparation, sequencing, and analysis. We thank K. de Bosscher (Ghent University) and J. Taipale (Karolinska Institutet) for providing plasmids. E.T. was supported by grants from the Center for Innovative Medicine (CIMED) at the Karolinska Institutet, the Swedish Research Council, the Swedish Cancer Society, the Swedish Diabetes Foundation, the Novo Nordisk Foundation, and the European Union FP7 HEALTH project HUMAN. R. F. was supported by the European Foundation for the Study of Diabetes (EFSD)/Lilly research fellowship, Junior Diabetes Wellness Sverige grant and KI research foundation grants. R.K. and T.S. were supported by KI/SL and grants from CIMED. N.L. received a doctoral education grant (KID) from Karolinska Institutet. Z.H. was supported by Chinese Government Scholarship (CSC). N.V. was supported by grants from the French National Agency of Research (CONRAD and PROVIDE), Region Ile de France (CORDDIM), Paris city (EMERGENCE), the French Foundation for Diabetes (SFD), and the European Union H2020 framework (ERC-EpiFAT 725790). The human liver transcriptome study performed by P.L., F.A., N.V., and B.S. was supported by EU [HEPADIP (Contract LSHM-CT-2005-018734) and RESOLVE (Contract FP7-305707)], the European Research Council (ERC Grant Immunobile, contract 694717), Fondation pour la Recherche Médicale (Equipe labellisée, DEQ20150331724) and Agence Nationale pour la Recherche (ANR-10-LBEX-46). B.S. is a recipient of an Advanced ERC Grant (694717). We thank all members of our laboratories for scientific discussions and materials.

\section{Author contributions}

R.F. and E.T. designed and supervised the study. N.L. and R.F. performed most experiments. A.D. and N.L. analyzed the NGS data. N.L., S.G., M.G., S.B., and R.F. performed the animal experiments. O.A., M.P., L.V., and P.P. analyzed the lipoprotein profiles and joined discussions. N.L., R.F., Z.H., and T.J. helped with the cloning and molecular experiments. A.M. and A.H. provided and processed the Ncor and Smrt KO livers. T.S. and R.K. analyzed the pathology. P.L., F.A., N.V., S.F., L.G., and B.S. provided and analyzed human data. R.F., N.L., and E.T. wrote the manuscript. All authors discussed the results and commented on the manuscript.

\section{Additional information}

Supplementary Information accompanies this paper at https://doi.org/10.1038/s41467 019-09524-z.

Competing interests: The authors declare no competing interests.

Reprints and permission information is available online at http://npg.nature.com/ reprintsandpermissions/

Journal peer review information: Nature Communications thanks the anonymous reviewer(s) for their contribution to the peer review of this work. Peer reviewer reports are available

Publisher's note: Springer Nature remains neutral with regard to jurisdictional claims in published maps and institutional affiliations.

Open Access This article is licensed under a Creative Commons Attribution 4.0 International License, which permits use, sharing, adaptation, distribution and reproduction in any medium or format, as long as you give appropriate credit to the original author(s) and the source, provide a link to the Creative Commons license, and indicate if changes were made. The images or other third party material in this article are included in the article's Creative Commons license, unless indicated otherwise in a credit line to the material. If material is not included in the article's Creative Commons license and your intended use is not permitted by statutory regulation or exceeds the permitted use, you will need to obtain permission directly from the copyright holder. To view a copy of this license, visit http://creativecommons.org/ licenses/by/4.0/.

(C) The Author(s) 2019 